\title{
Civilisations
}

Revue internationale d'anthropologie et de sciences

humaines

52-1 | 2004

Transmission des savoirs et interactions culturelles

\section{Kuju/kwap, kide/too : La valence des coordonnées spatiales de la topographie Turkana}

\section{Pierre Liénard}

\section{OpenEdition \\ Journals}

Édition électronique

URL : http://journals.openedition.org/civilisations/744

DOI : 10.4000 /civilisations.744

ISSN : 2032-0442

\section{Éditeur}

Institut de sociologie de l'Université Libre de Bruxelles

\section{Édition imprimée}

Date de publication : 1 mai 2004

Pagination : 81-113

ISSN : 0009-8140

\section{Référence électronique}

Pierre Liénard, «Kuju/kwap, kide/too : La valence des coordonnées spatiales de la topographie Turkana », Civilisations [En ligne], 52-1 | 2004, mis en ligne le 27 mai 2009, consulté le 02 juin 2020. URL http://journals.openedition.org/civilisations/744 ; DOI : https://doi.org/10.4000/civilisations.744 


\section{Kuju/kwap, kide/too : \\ La valence des coordonnées spatiales \\ de la topographie Turkana}

\section{PIERRE LIÉNARD}

« [...] les sociétés aménagent leur environnement en fonction de l'interprétation qu'elles en font, et réciproquement elles l'interprètent en fonction de l'aménagement qu'elles en font. " (Augustin Berque, 1995, p.15)

Dans cette contribution, je m'attache à déterminer la valence " générique » que certaines composantes de l'espace (tel qu'il est vécu, appréhendé et conçu par les agents) acquièrent dans l'univers culturel turkana. Le terme de «valence » demande quelques éclaircissements. J'utilise ce terme sous sa définition psychologique. La valence exprime la puissance de l'attrait ou, plus précisément, le degré d'attraction qu'un sujet individuel, une activité ou un objet exerce en tant qu'objectif comportemental pour un individu ou un groupe d'individus.

Pour construire mon argumentation, j'utilise deux sources de données. Premièrement, j'observe le conditionnement écologique de la région où furent menées les enquêtes ethnographiques et ses effets sur les modalités de l'exploitation du territoire dans le cadre d'une économie pastorale. Cette première approche me permet de proposer des hypothèses sur les conséquences vraisemblables de ce conditionnement pour l'élaboration des représentations indigènes de l'espace. Deuxièmement, par l'analyse de l'organisation spatiale et des mouvements des participants de deux procédures rituelles, je présente succinctement les valeurs attribuées, dans ces activités conventionnelles collectives, à certaines composantes de l'espace.

Je parle donc de la topographie telle qu'elle est représentée et conceptualisée par les membres d'une population pastorale et guerrière du Nord-Ouest Kenyan, les Turkana. Dans l'argumentation, je défends successivement deux hypothèses générales.

1. L'appréhension indigène primaire de l'espace est fortement déterminée par les conditions écologiques extrêmes de la région que la population turkana occupe,

- par le modèle économique prédominant d'utilisation du territoire,

- par l'environnement ethnique dans lequel s'insère la population.

2. L'acquisition des «descriptions » de l'espace se réalise dans des contextes distincts (bien qu'en relation étroite) :

- au « quotidien », dans le cadre de l'économie pastorale,

- lors de l'exposition répétée à la matrice spatiale des rituels sacrificiels. 
Je mets en évidence le rapport échö̈que qui existe manifestement entre les descriptions rituelle et ordinaire. Les représentations de l'espace que se donnent les agents semblent être ainsi profondément structurées par ces deux univers pratiques que sont le rituel et le pastoralisme. Les différentes «modélisations » rituelles de l'espace participent donc aussi à la détermination et au renforcement de la valeur des concepts spatiaux. Cette influence sur la valeur se produit, entre autre, lors du (re-)cadrage rituel imposé de manière récurrente aux événements marquants de la vie de l'individu ou du groupe. L'analyse comparative de la spatialisation des actions rituelles accomplies dans des contextes différents me permet d'aborder ce questionnement.

En conclusion, je soulève le problème de l'induction, par le façonnement des affects, de dispositions particulières chez les acteurs du rituel. C'est, à mon sens, dans ce domaine spécifique que s'exerce pleinement l'efficacité du rituel. Bien sûr de l'information explicite est acquise mais elle constitue une partie seulement de ce qui est véritablement communiqué. De fait, la transmission interindividuelle (et, plus généralement, entre les membres de différentes catégories sociales) d'informations spécifiques sur les différentes composantes (et sur leurs valeurs) de l'espace turkana est généralement sous-déterminée par la procédure rituelle. Celle-ci ne peut donc être assimilée à une forme particulière de discours dont l'observateur pourrait immédiatement extraire des propositions sur le monde.

L'action rituelle permettrait plutôt une évocation, par la présentation et par la manipulation « réaliste » d'indices que, à la suite de Charles S. Pierce, Alfred Gell définit comme des entités à partir desquelles l'observateur peut faire des inférences causales d'un certain type ou des inférences à propos des intentions ou des capacités d'une autre personne (Gell 1998 : 13). Il ne semble donc pas y avoir de langage rituel dans un sens strict même si, dans l'action rituelle, les participants peuvent recourir à l'emploi d'éléments linguistiques. Par contre, la procédure rituelle concourt très certainement à instiller implicitement, dans l'esprit des participants, des habitudes cognitives, des dispositions et, en définitive, des croyances de bas niveau qui conditionneront ultérieurement la conduite.

\section{ÉLÉMENTS ETHNOGRAPHIQUES}

Dans les développements qui suivent, je traite succinctement des caractéristiques générales du système social de la population turkana. Je ne m'attarde pas sur les débats (par ailleurs très importants) qui touchent à la détermination de la valeur exacte de certaines des institutions (générations, classes d'âge) présentes dans cette population. Ce n'est pas là le sujet de mon exposé. Plusieurs auteurs se sont déjà penchés sur ces questions. Le lecteur désireux de plus amples informations s'y reportera (Gulliver 1951, 1958 ; Müller 1989, 1991).

La population turkana occupe un territoire au Nord-Ouest du Kenya, dans un espace limitrophe de l'Ouganda, de l'Ethiopie et du Soudan. Elle constitue le plus important groupe ethnique de la région (approximativement 250000 âmes). Les Turkana font partie du groupe linguistique des Paranilotes (anciennement dénommés Nilo-Hamites) et, plus précisément, d'une de ses deux branches, la branche orientale encore appelée la branche des Nilotes des plaines. 


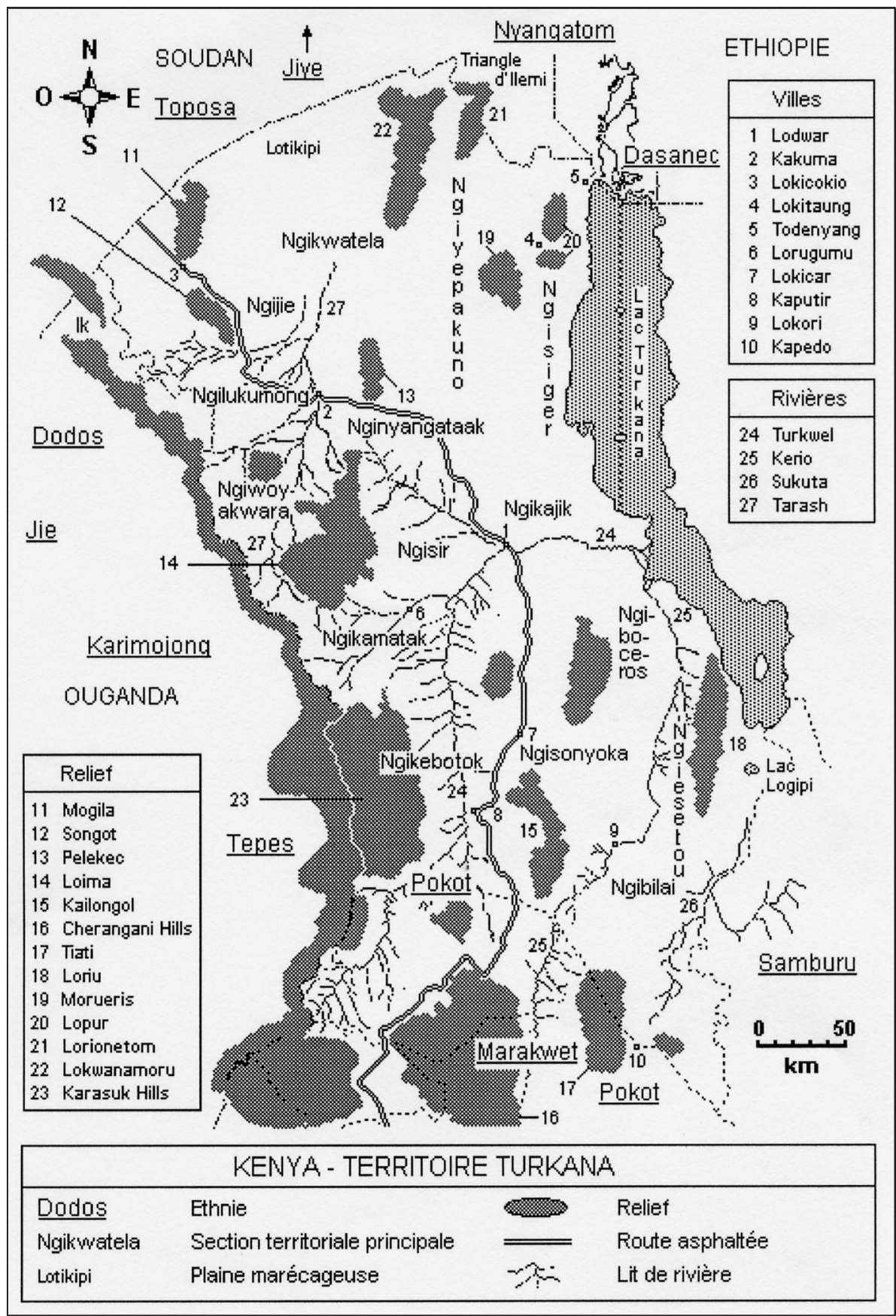

Fig. 1 
La branche des Nilotes des plaines comprend trois sous-groupes linguistiques : celui du Nord dont l'ethnie Bari fait partie, celui du Sud auquel appartiennent les Maasaï et celui du centre qui groupe huit ethnies, les Karimojong, les Jie, les Dodos, les Toposa, les Jiye, les Nyangatom, les Turkana et les Teso. Sept ethnies de ce groupe central sont regroupées sous l'appellation de Karimojong Cluster. Elles se partagent des territoires qui bordent ou chevauchent, pour certaines, des frontières internationales. Ce sont les Karimojong, les Jie et les Dodos des franges Est de l'Ouganda, les Toposa et les Jiye du Sud Soudan, les Nyangatom de la vallée de l'Omo en Ethiopie et du Sud-Est soudanais, et les Turkana du Kenya.

Les ethnies du Karimojong Cluster occupent le cœur d'une région qui a connu pendant de nombreuses années des troubles graves. La guerre civile a fait rage en Ouganda et en Ethiopie avant que ne viennent la stabilisation et la démocratisation de leurs régimes politiques respectifs dans les années 1990. Les populations du Sud Soudan sont encore actuellement impliquées dans une guerre civile dramatique ${ }^{1}$. Une des conséquences de cette longue instabilité régionale (passée ou présente) est observable dans le surarmement généralisé des populations pastorales de la zone de frontières internationales. Certaines ethnies, comme les Toposa, les Nyangatom, les Karimojong, les Dodos et les Pokot, bénéficient d'un accès privilégié aux sources d'approvisionnement en armement moderne (principalement des fusils d'assaut AK47, G3, FN)². Cette disparité d'accès entraîne une nouvelle redéfinition de l'étendue des territoires de certaines ethnies. On peut observer, par exemple, que les populations nyangatom et toposa se sont complètement appropriées des territoires qu'elles ne contrôlaient, semble-t-il, que lâchement il y a de cela 20 à 30 ans (au tout début de la prolifération des armements modernes dans la région) ${ }^{3}$.

L'existence de frontières internationales n'est pas sans conséquences pour l'utilisation traditionnelle de l'espace. Si l'on observe attentivement la situation sur les franges Ouest du Turkan, on peut remarquer que les derniers pâturages où les

1. Depuis l'indépendance de leur pays en 1956, les populations du Soudan ont vécu une seule interruption de longue durée des conflits internes, de 1972, fin de la première guerre civile, à 1983, début de la seconde guerre civile.

2. Ces sources sont des plus diverses. Les armes sont issues de la contrebande interne aux pays ou du trafic inter frontalier. Les circuits de distribution sont très intriqués et des ennemis de toujours s'approvisionnent souvent aux mêmes sources. Un exemple donnera une idée de l'ampleur du trafic. Une bonne partie des munitions présentes dans le Nord Turkan sont de fabrication soudanaise (du Nord). Ces munitions doivent manifestement transiter par des territoires ennemis avant de parvenir dans le pays turkana: au Soudan, entre le Nord et le Sud et, entre Soudan et Kenya, des territoires toposa aux territoires turkana. La présence des troupes militaires de tous bords (FPLA, armée soudanaise du Nord) dans le Sud Soudan facilite grandement la circulation des armes. D'autre part, de chaque côté des frontières ethniques, des individus se sont fait la spécialité d'acheminer les biens de contrebande (dont font partie les armements).

3. Les Toposa et les Nyangatom empièteraient de plus en plus sur toute l'étendue des marges nord de leurs territoires. La situation des Nyangatom est assez particulière. La zone centre - Ouest de leur pays, le triangle d'Ilemi (qui est officiellement dans le territoire soudanais) est maintenant sous contrôle kenyan. L'accès aux pâturages de cette région qu'utilisaient habituellement les Nyangatom pour y faire paître leurs troupeaux leur est régulièrement interdit par les autorités régionales kenyanes afin de limiter les risques de conflit entre guerriers turkana et nyangatom. La « poussée » vers le Nord pourrait donc aussi être, en partie, la conséquence de la suppression de l'accès aux pâturages du triangle d'Ilemi. 
Turkana mènent leurs troupeaux au cœur de la saison sèche se trouvent à l'intérieur de l'Ouganda, bien au-delà de la frontière internationale. C'est dans ces situations critiques que l'on peut constater que les deux logiques territoriales - nationale et pastorale - coexistent parfois difficilement. Ce qui, pour les Turkana, constitue une migration nécessaire, voire même impérieuse si l'on veut éviter la décimation des troupeaux, représente, pour les autorités ougandaises, une intrusion massive de kenyans sur son territoire.

De même des raids ennemis en territoire turkana sont souvent investis d'une dimension internationale qu'ils ne devraient, somme toute, pas avoir. Une partie du problème réside dans la volonté d'attribuer à ses populations une identité nationale qui s'articule parfois difficilement avec les nécessités du pastoralisme dans ces régions arides. En effet, l'appartenance nationale des populations de la région est définie par la localisation de leur centre ethnique sans que ne soient prises en compte les possibilités de « débordements » que la nécessité peut imposer (lors d'une sécheresse sévère, par exemple). Les mouvements pastoraux et guerriers transfrontaliers perdent ainsi leur caractère normal dans l'économie pastorale et enveniment parfois les relations régionales des Etats nations de cette partie de l'Afrique.

Les ethnies du Karimojong Cluster présentent un faciès agro-pastoral avec, pour certaines d'entre elles, un renforcement d'un des deux pôles (agricole et pastoral) à des périodes spécifiques de l'année. La société turkana repose, cependant, plus fortement sur l'économie pastorale, étant donné l'extrême aridité du Turkan (le territoire turkana). Lorsque les pluies sont abondantes, de petites cultures de période de crue (principalement du sorgho) sont mises en place aux abords des lits des rivières. Les Turkana élèvent des zébus, des dromadaires, des chèvres, des moutons et des ânes.

La société turkana est divisée en deux générations (anaket, qui désigne aussi la classe d'âge) : celle des Montagnes (Ngimoru) et celle des Léopards (Ngirisai). Un principe fondamental régule la succession de ces générations: un enfant naît dans la génération alterne à celle de son père. L'appartenance générationnelle n'est pas pertinente pour le sexe féminin. Cependant, une fois mariée, la femme doit porter un collier de mariage métallique (alagama) de couleur jaune (cuivre, laiton) si son mari appartient à la génération des Léopards et gris (fer) si son mari est Montagne. Il semble donc y avoir une sorte d'alignement du statut de la femme sur celui de son mari. Les générations se partagent en diverses classes d'âge (anaket) successives entre lesquelles se répartit la population masculine.

La constitution des classes d'âge se conforme à un processus graduel de reconnaissance collective sanctionnée par l'asapan, une procédure rituelle à laquelle nous pourrions attribuer généralement le statut d'initiation (elle est, en fait, plus exactement, la confirmation/sanction d'une évolution). Dans les sections territoriales du Nord Turkan, où j'ai principalement mené mes enquêtes ethnographiques, le recrutement se fait simultanément et de manière indépendante en fonction de l'appartenance à l'une des deux générations alternes. Il y a donc deux séries indépendantes de classes d'âge successives : l'une se rattachant à la génération des Montagnes, l'autre à la génération des Léopards. Les séries de dénominations des classes d'âge diffèrent selon la section territoriale envisagée.

La population se répartit en deux groupements territoriaux: celui des Ngicuro (de acurur, pl. ngacuro, le canyon, le torrent encaissé, " ceux des torrents »), qui occupe approximativement les régions de l'Ouest du Turkan, et celui des Ngimonia 
(de amoni, pl. ngamoni, la forêt, la brousse dense, " ceux des forêts »), qui occupe approximativement les régions de l'Est et du centre. Ces deux entités se subdivisent en sections territoriales (ekitela), attachées chacune à un territoire précis.

Le territoire d'une section comprend les pâturages de saisons des pluies (qui représentent l'ere véritable, le « cœur du terroir »), au moins un tronçon du lit d'une rivière importante ${ }^{4}$ et une zone montagneuse dans et à proximité de laquelle sont localisés des pâturages de saison sèche $e^{5}$. La section territoriale « se pense » comme une unité économique, défensive et culturelle relativement autonome. Chaque section possède en effet son style (enape) vestimentaire féminin caractéristique et, comme je l'ai déjà signalé, ses séries de noms de classes d'âge masculines ${ }^{6}$. La section présente manifestement une tendance à l'endogamie. Ce n'est cependant pas en raison d'une règle spécifique qui prohiberait les alliances avec des femmes d'autres sections. De nombreux couples réunissent, en effet, des individus de sections différentes. Ce sont plus sûrement les stratégies d'alliance gouvernant partiellement les choix matrimoniaux qui expliquent cette tendance à l'endogamie des groupes régionaux. Je peux l'exprimer de façon caricaturale. En se mariant dans un groupe qu'il côtoie, un homme est certain de pouvoir bénéficier périodiquement de ses relations d'alliance avec les membres de la parentèle de l'épouse. Par contre, s'il épouse une femme d'une section éloignée, une fois les cérémonies de mariages accomplies, il sera beaucoup plus difficile d'en appeler aux parents de l'épouse restés dans le territoire de leur section (la résidence est patrilocale). L'époux perd ainsi une bonne partie des bénéfices de l'alliance entre les deux familles que scelle l'union du couple.

Et, finalement, la société turkana est encore partagée en clans (emacar, «marque » pyrogravée sur le bétail) exogamiques qui se répar-tissent inégalement dans les différentes sections territoriales qui se partagent le Turkan. Un enfant naît dans le clan de son père (filiation patrilinéaire) si la compensation matrimoniale a été versée par la famille de celui-ci. La femme, à son mariage, est intégrée rituellement dans la parentèle et dans le clan de son époux (ce qui n'implique pas une oblitération complète de la provenance clanique de la femme $)^{7}$.

4. Etant donné le besoin d'une grande quantité d'eau pour le bétail, le bassin d'une rivière doit toujours être accessible. La majorité des migrations se fait donc par rapport à un lit de rivière et le déplacement est généralement qualifié de « vers l'amont » / akilot kuju (« aller amont ») ou « vers l'aval » / akilot kwap («aller aval »). Je reparle de cette problématique lorsque je traite des axes qui « médiatisent» la perception de l'espace dans l'univers culturel turkana.

5. Les populations du centre et du Sud utilisent aussi des pâturages de saison sèche situés à proximité du lac Turkana dans les deltas de la Kerio et de la Turkwell.

6. Les sections territoriales ont aussi, chacune, leurs lieux d'initiation masculine (asapan).

7. La jeune mariée doit respecter un grand nombre d'interdits (habillement, coiffure,...) que toutes les jeunes mariées du clan qu'elle rejoint doivent suivre. Les rituels, règles et interdits liés à la naissance que doit respecter la mère sont ceux du clan du mari. J'ai, cependant, rencontré plusieurs situations dans lesquelles une femme suivait un interdit qui, elle l'affirmait, provenait du clan de son père (ou, selon les dires de mon informatrice, avait été prescrit, il y a longtemps, par un devin, à des membres du clan de son père). Ainsi, l'intégration dans le clan du mari et l'effacement de la provenance, pour réels qu'ils sont (une femme mariée interrogée sur sa provenance clanique répondra immanquablement par l'énonciation du non du clan de son époux), ne gomment pas entièrement l'identité clanique de jeune fille de la femme. 
Le district du Turkana couvre approximativement une superficie de $65000 \mathrm{~km}^{2}$ dans le Nord-Ouest du Kenya entre les $34^{\circ}$ et $37^{\circ}$ méridiens et entre $1^{\circ}$ et $5^{\circ}$ de latitude nord (Müller H. K. 1989 : 22). Le territoire est ceinturé de chaînes de montagnes. A l'Ouest, s'élèvent les contreforts ougandais qui se terminent au Sud du Turkan par les Karasuk Hills et par les Cherangani Hills. Accompagnant le tracé de la vallée de la Sukuta, les Samburu Hills délimitent la frontière Sud du territoire. Au Nord, d'ouest en est, quatre chaînes de montagnes égrènent le paysage : la Mogila Range, séparée des trois autres massifs du Nord-Est par la grande plaine marécageuse de Lotikipi, la Lokwanamoru Range, la Lorionetom Range et la Lopur Range. A l'Est, le territoire turkana est limité par le grand lac Turkana (anciennement Lac Rudolph).

Le cœur de l'espace turkana est composé d'une plaine déclive d'ouest (altitude $800 \mathrm{~m}$ ) en est ( $375 \mathrm{~m}$, altitude du lac Turkana) ce qui explique l'orientation générale du drainage des eaux (Sud-Ouest/Nord-Est). Quatre grandes rivières (et leurs affluents) partagent le Turkan. Deux seulement sont permanentes les années pluvieuses et semi-permanentes les années de sécheresse : la Kerio et la Turkwel, qui traversent respectivement le Sud du district, au départ des Cherangani Hills, et le centre du pays, au départ des West Pokot, pour se déverser dans le lac Turkana. Les deux autres rivières importantes, non permanentes celles-ci, alimentent, en période des pluies, pour la Tarash, les grandes plaines et les marais du Nord (Lotikipi), pour la Sukuta, le lac Logipi au sud du lac Turkana. D'autres rivières de moindre taille s'écoulent des ensembles montagneux (ou collinaires) situés dans le Turkan et sur son pourtour. Elles ne sont alimentées en eau que très brièvement lorsque des pluies occasionnelles frappent les massifs dans lesquelles elles prennent leur source.

Les Turkana divisent l'année (ekaru) en deux saisons distinctes : la akiporo ou saison des pluies et la akamu ou saison sèche. L'année climatique "normale » est donc constituée de deux blocs saisonniers que je pourrais situer approximativement dans le temps d'avril à septembre pour la akiporo et d'octobre à mars pour la akamu. Remarquons, sans entrer dans les détails, que ce cycle varie fortement selon les années. Très souvent la période de sécheresse se prolonge jusque tard dans ce qui normalement devrait être la saison des pluies et, inversement, des épisodes pluvieux importants surgissent parfois au cœur de la saison sèche. Un usage linguistique particulier des termes akiporo et akamu souligne cette relative indétermination des cycles saisonniers. Akiporo peut généralement s'utiliser pour désigner une période pendant laquelle les pâturages sont verdoyants et $a k a m u$, un épisode climatique pendant lequel les pâturages sont brûlés par le soleil, sans qu'il ne soit pour autant fait référence aux saisons proprement dites (i.e. d'années «normales »).

Ce conditionnement écologique contraignant, dont est issue l'appréhension de l'écoulement du temps en termes généraux d'alternances entre deux saisons climatiques contraires, a encore des effets importants sur la catégorisation turkana des composantes du territoire. La plaine centrale du Turkan recèle peu de ressources hydriques permanentes. L'occupation prolongée de cet espace est donc possible uniquement lorsque les précipitations sont abondantes. En dehors de ces périodes, la quasi-totalité de la population et des troupeaux se retrouve aux abords de la frange montagneuse ougandaise et des autres chaînes de collines dans le Nord et le Sud du Turkan où se situent les pâturages de saison sèche. Une grande partie de l'année, le cœur de l'es- 
pace turkana est donc évacué par la majorité de sa population. Je parle d'une tendance générale. Pour être plus spécifique, je devrais traiter de la localisation dans l'espace du Turkan des différentes catégories de bétail. Les camélidés, les caprins et les ovins restent plus longtemps dans la plaine que les bovins. Ces derniers nécessitent un approvisionnement régulier en eau. De ce fait, ils pâtureront les plaines centrales pour une courte période lorsque les précipitations sont abondantes et soutenues.

Cette oscillation saisonnière entre montagnes et plaines, rendue nécessaire par le manque de précipitations régulières et par l'absence concomitante de ressources hydriques et de pâturages pour le bétail, donne naissance à deux types de sociabilité distincts, selon l'espace occupé. Les régions montagneuses du pourtour du Turkan correspondent à des frontières ethniques. Or, les Turkana entretiennent des relations conflic-tuelles avec la majorité des ethnies limitrophes : au nord avec les Dasanec, les Nyangatom et les Toposa, au sud, principalement avec les Pokot et les Tepes, à l'ouest avec les Dodos et les Jie. D’autres ethnies, plus éloignées des régions de conflits, subissent dans une moindre mesure les incursions turkana. Les activités guerrières prennent, généralement, la forme de razzias en territoire ennemi pour y voler le bétail.

La menace permanente de l'irruption d'ennemis exerce une grande influence sur le patron de l'habitat des populations de part et d'autre de ces frontières ethniques. Les campements nomades (adakar, « la communauté de pâturage ») turkana des régions montagneuses sont compacts et rassemblent une population nombreuse ${ }^{8}$. Chaque camp est en relation étroite avec les autres camps de la région. La stratégie recherchée est de présenter un front commun face à l'ennemi. Les hommes des différents camps d'une même région se concertent régulièrement pour décider tout déplacement, plus avant en territoire adverse ou vers les arrières.

Cette période de l'année, pendant laquelle la majorité de la population occupe les franges Ouest du territoire, est ainsi fortement marquée négativement ${ }^{9}$. Plusieurs éléments se combinent pour créer un climat d'extrême tension au sein de la population. La mobilité générale est réduite en raison des risques de rencontre inopportune avec des maraudeurs ennemis. La cohabitation forcée dans de grands ensembles limite l'in-

8. Selon mes observations, les grands campements peuvent compter approximativement 250 à 300 personnes. Exceptionnellement, les populations de plusieurs communautés de pâturages se réunissent pour un temps et constituent de très grands campements. Ces campements sont appelés arigan vraisemblablement de la racine verbale -rig qui désigne l'action de débarrasser, par frottement/arasement, quelque chose de son couvert. Le terme fait référence à l'aspect général du paysage autour de ces campements: le sol est « propre », débarrassé de tout végétal, et les arbres avoisinants ont été élagués (leur bois a servi de combustible).

9. Dans le Nord-Est du Turkan, une partie de la population (des sections territoriales Ngiyepakuno et Ngissiger) et du bétail occupe, en saison sèche, les régions avoisinant les collines Lorionetom et Lokwanamoru, situées au nord des pâturages de saison des pluies. A nouveau, ces zones refuges de saison sèche sont exposées aux populations ennemies. Ce type de migration (vers le nord) est une exception dans l'espace du Turkan. Ceci dit, une bonne partie du gros bétail de ces populations (hormis les dromadaires qui supportent des conditions très dures) réside à l'année aux abords des montagnes de l'Ouest du Turkan. Les migrations dans l'extrême Sud-Est du Turkan sont elles aussi particulières. Je ne rentre pas ici dans les subtilités du gardiennage, de la localisation des troupeaux et de l'interpénétration des zones de pâturages « refuges » des différentes sections territoriales. Je devrais introduire trop de détails, non-pertinents dans l'argumentation que je propose, pour que le lecteur puisse comprendre l'ensemble des circuits de migration. A l'échelle du pays turkana, il n'est pas faux d'affirmer, globalement, que lorsque la saison sèche arrive, il est temps de regagner les territoires de l'Ouest montagneux (ou plus exactement: les territoires à l'ouest d'où l'on se trouve!). 
timité à son minimum. Les pâturages et les sources d'eau s'épuisent progressivement sans autre recours possible que de s'avancer plus profondément dans les montagnes et donc de se rapprocher encore plus de l'ennemi. Du fait de la mauvaise alimentation du bétail, le lait, source principale de subsistance, fait défaut et on doit se résoudre à abattre des animaux pour pallier ce manque d'approvisionnement.

En complète opposition avec cette atmosphère « tendue », nous trouvons le mode de sociabilité de plaine. On se retrouve au cœur de l'ere, « le pays », « le chez-soi ». L'ennemi est à bonne distance (même si cela n'écarte pas toute menace de raid). Les communautés de pâturages sont beaucoup plus dispersées. La mobilité générale est accrue. C'est le temps de la socialisation « joyeuse ». Les amis se rendent visite. Les hommes courtisent les jeunes filles. Des arrangements matrimoniaux sont conclus entre familles. Des mariages sont célébrés. Les jeunes gens exécutent de nombreuses danses à la tombée du jour. Avec le retour des pluies, les activités pastorales sont généralement facilitées. La fertilité retrouvée des pâturages écourte la durée et diminue la fréquence des déplacements des troupeaux. Du temps libre se dégage donc. L'eau des puits est facilement accessible et ne manque pas. La nourriture est abondante. La proximité des centres urbains localisés dans la plaine permet, grâce au troc d'animaux et de lait, de varier les sources d'alimentation, plus facilement qu'en saison sèche.

Il est intéressant de remarquer que la « donne » urbaine - qui est un fait marquant de la plaine centrale du Turkan - est généralement bien intégrée dans les économies pastorales régionales. Les centres « urbains » (agglomérations, centres administratifs, postes de traite et/ou canton-nements militaires) exercent une attraction manifeste sur les populations qui nomadisent encore. On y trouve des denrées qui, ces dernières années, semblent être devenues indispensables dans l'économie nomade turkana. Je pense plus spécifiquement à la farine de maïs et au sucre. Très généralement, on peut affirmer que les agglomérations représentent actuellement une source d'approvisionnement non-négligeable. Les installations des campements en plaines qui se font toujours en fonction des ressources hydriques et des pâturages disponibles prennent ainsi souvent en compte, maintenant, les possibilités d'accès aux ressources des centres urbains. Notons que ces centres sont fréquemment situés dans des endroits qui, traditionnellement, étaient des lieux de passage obligé (une zone de puits semi-permanents, une région accolée à un bassin de rivière, une voie de pénétration naturelle dans un zone de pâturages saisonniers,...). Ces localisations stratégiques (occupées pour faciliter le contrôle du territoire et de ses populations) rendent les centres urbains accessibles, en saison des pluies, pour la quasi-totalité des groupes locaux et régionaux.

On remarque, d'autre part, une volonté affirmée de la part des groupes familiaux d'être présents de manière quasi-permanente, par l'intermédiaire d'un ou de plusieurs de ses membres, dans les agglomérations, ceci, afin de bénéficier des ressources qui s'y trouvent. Le milieu urbain et ses environs immédiats fournissent, en effet, de nombreuses opportunités économiques. Dans le milieu urbain, les familles trouvent une variété de substituts aux moyens traditionnels de subsistance ${ }^{10}$. Des aides alimentaires y sont distribuées par les Églises, par les O.N.G. ou par l'Etat. On peut se rabattre momentanément sur des micro-activités telles que la production d'alcool clandestin ou

10. Lorsque les temps sont durs et que la sécheresse décime les troupeaux, les agglomérations et leurs extensions (les villages permanents et semi-permanents alentours) connaissent une explosion de leur po- 
l'artisanat à destination des populations pastorales de l'arrière-pays (ferronnerie,...).

Les agglomérations exercent encore une autre influence observable sur les patrons d'occupation de l'espace. Tous les membres d'un groupe familial résident très rarement au même endroit. Ils se répartissent le plus souvent entre la résidence de la awi napolon, littéralement « la grande maison »- le campement où demeurent le(s) doyen(s) de la maisonnée, ses (leurs) femmes et enfants - et les différentes awi a$a b o r$, les résidences satellites où vivent les autres membres de la « maisonnée »/awi (comprise comme l'unité sociale dépendante d'un pater). Si le groupe familial se scinde de la sorte c'est pour des questions de gestions du bétail et d'utilisation optimale du terroir. On remarque qu'avec l'âge, les doyens des groupes familiaux choisissent de déplacer beaucoup moins souvent la awi napolon et, finalement, de l'installer pour de très longues périodes aux mêmes endroits, proches de l'un ou l'autre centre urbain. Dans ces campements, ne se trouvent que quelques rares animaux dont une majorité d'ovins et de caprins qui supportent relativement bien le manque d'eau et de pâturages verdoyants. Ainsi, tout autour des centres urbains (dans un diamètre de plusieurs dizaines de kilomètres), sont installées, pratiquement toute l'année, de nombreuses vieilles personnes en compagnie d'une partie de leur parenté. Même au cœur de la saison sèche, la awi napolon reste constamment en contact avec les awi a-abor qui lui fournissent régu-lièrement des animaux en période de lactation et reprennent les autres animaux épuisés par leur séjour dans la plaine aride pour leur faire reprendre des forces dans de meilleurs pâturages. L'existence des centres urbains semble bien avoir facilité ce genre d'occupation de l'espace. Sans possibilité de se procurer en « ville » divers aliments de substitution, il serait, en effet, très difficile d'occuper la plaine du Turkan en saison sèche.

Nous avons vu se dessiner une polarité entre un espace très contraignant et un espace relativement moins contraignant (où l'influence des centres urbains se fait sentir de diverses manières). Ma présentation s'est voulue schématique pour distinguer clairement ces deux pôles. Bien évidemment, il existe un grand nombre de situations intermédiaires. Mais, je peux affirmer que les deux représentations des modes distincts d'appréhension de l'espace dont je viens de tracer les grandes lignes sont généralement véhiculées dans le discours des membres de la société turkana.

\section{L'AMONT, L'AVAL, L'OUEST ET L'EST : UN ESPACE ORIENTÉ}

Deux axes directionnels fondamentaux déterminent l'ensemble des représentations turkana de l'espace. Le premier de ces axes est calqué sur la course du soleil

pulation. Les uns s'y installent de manière définitive, les autres y vivent le temps de pallier le manque de ressources momentané pour reprendre ensuite la vie pastorale. Pour faire face aux difficultés passagères ou pour se ménager des passerelles vers le monde de la modernité, certains parents placent leurs enfants dans un des pensionnats partiellement subventionnés par l'Etat kenyan et par les différentes Églises présentes dans le Turkan. Cette démarche rentre dans la logique pastorale générale de multiplier l'origine des ressources. Le petit bétail pâture dans une région, les camélidés dans une autre, les bovins dans une troisième. Une partie des membres de la maisonnée s'occupe de la gestion des troupeaux, une autre des micro-cultures là, où et quand c'est possible et un troisième groupe entretiendra une relation privilégiée avec l'univers urbain pour y bénéficier d'éventuelles opportunités. 
d'est (kide) en ouest (too). L'autre se rapporte aux cours des rivières, de l'amont ( $k$ uju) vers l'aval (kwap), qui dans le Turkan suivent approximativement un axe sud-ouest/ nord-est (avec, bien sûr, des variations locales et régionales). Dans le quotidien pastoral, il est régulièrement fait appel à ces deux axes pour orienter l'action. Les troupeaux nécessitent des pâturages mais aussi de grandes quantités d'eau. C'est pourquoi l'ensemble des migrations se fait le long (ou à proximité) de bassins de rivières dans les lits desquelles on pourra creuser les puits. L'orientation générale du déplacement des campements et des troupeaux sera donc très souvent spécifiée en terme de mouvement vers l'amont (akilot kuju) ou vers l'aval (akilot kwap).

Plus généralement encore, pour l'ensemble des déplacements (même sans troupeau), lorsqu'un Turkana cherche à indiquer un chemin il s'efforce de se référer à des lits de rivières pour décrire la route à parcourir. Même lorsqu'il s'agit de se déplacer vers une destination que nous situerions au Nord ou au Sud, aucun problème de détermination de l'orientation à suivre ne se pose. A partir d'un lieu-dit connu de l'inter-locuteur ou à partir de l'endroit où se produit la communication, il donnera la direction générale de déplacement jusqu'à ce qu'une rivière soit rencontrée et ainsi de suite, nommant successivement les rivières et les zones de pâturages intercalaires. Pour comprendre cette affirmation, il faut se rappeler que la plaine centrale du Turkan est interrompue régulièrement de lits de ruisseau/rivière ${ }^{11}$ qui dévalent des massifs montagneux (ouest/sud-ouest), courent parallèlement (en direction de 1'est/nord-est) et délimitent ainsi des zones de pâturages précises. Localement (aux abords des massifs du Nord du Turkan, par exemple), l'orientation des lits de rivière peut se modifier fortement, voire s'inverser, mais, globalement, à l'échelle du territoire turkana, l'orientation de la majorité des cours d'eau importants se conforme au schéma présenté. Pour déterminer les changements éventuels d'orientation dans le déplacement, l'interlocuteur se référera à nouveau au sens d'écoulement des cours d'eau ou à l'axe kide/too lorsque le déplacement ne se fait pas directement par rapport au lit d'une rivière.

L'axe kide/too que suit la trajectoire du soleil est donc aussi utilisée pour déterminer l'orientation des déplacements ou la position dans l'espace de quelque chose (une habitation par rapport à une autre, un emplacement précis par rapport au lieu où l'on se trouve,...). D'une manière générale, lorsque le locuteur ne se réfère pas à un lit de rivière, il peut toujours recourir aux coordonnées absolues kide, too pour, approximativement, situer un lieu ou orienter un déplacement. Si j'insiste sur l'aspect approximatif de l'orientation c'est que dans l'usage courant kide comme too ne désignent pas des directions précises (l'est et l'ouest exacts d'une boussole) mais des zones comprises dans un angle plus ou moins ouvert dont le sommet est occupé par le locuteur ou un point de référence choisi. La mention de détails de l'environnement, un arbre remarquable, un lieu de passage du bétail, une zone de pâturage, les collines

11. J'ai affirmé qu'il n'y avait que 4 grandes rivières. Ce n'est pas faux. Cependant, l'espace du Turkan est aussi sillonné de lit de ruisseaux / rivières (en fait des dépressions qui collectent les eaux) qui coulent exclusivement quand les pluies s'abattent sur les zones où ils prennent leur source. L'eau récoltée dans ces lits est évacuée dans des rivières plus grandes ou s'évanouit dans les sables selon les aléas du relief. Il subsiste toujours pour un temps de l'eau dans des poches rocheuses souterraines. Ces points d'accumulation et de retenue d'eau sont bien connus. Les quantités d'eau sont, cependant, rarement suffisantes pour permettre une utilisation intensive et très longue de ces réserves. L'approvisionnement en eau d'un camp se fait donc généralement à partir de plusieurs sources. 
(phénomènes naturels fortement saillants dans la plaine du Turkan)... permettra de préciser la localisation du lieu recherché.

Ceci étant dit, certains éléments de l'espace ne nécessitent pas d'être localisés précisément pour être trouvés. De nombreux indices de présence humaine dans l'environnement permettent de retrouver immédiatement une habitation, par exemple, et cela même si le voyageur n'avait été que très sommairement informé sur son emplacement (« c'est à kide! »). L'état de la végétation, les chemins menant aux puits et les circuits suivis par les animaux (qui finissent par marquer fortement un espace de plus en plus éloigné de la résidence) sont parmi les indices les plus facilement repérables.

La trajectoire du soleil est aussi utilisée pour mesurer les temps de déplacement. Un Turkana renseignera un interlocuteur sur la durée de marche d'un parcours en indiquant de la main (bras tendu vers le ciel, main en position horizontale par rapport au sol, face tournée vers le bas) l'endroit où se trouvera le soleil à la fin du déplacement. Bien sûr, il existe aussi un ensemble d'expressions linguistiques idiomatiques pour déterminer plus ou moins précisément des moments du jour ou de la nuit. Comme, par exemple, acaunet de acaun, «briller », qui désigne le moment auquel le soleil commence à scintiller, vers 9-10 heure du matin.

A un niveau d'abstraction plus élevé, l'axe too/kide sert encore à classer la population turkana. Les sections territoriales sont, en effet, réparties entre deux grands ensembles : " les gens de l'Ouest» (ngitunga alo too) et " les gens de l'Est et du centre » (ngitunga alo kide ka alo kiding). Les noms de ces deux groupements nous donnent un indice linguistique du lien générique avec un type d'environnement spécifique que les Turkana reconnaissent à chacune des deux coordonnées. «Les gens de l'Ouest » appartiennent à l'ensemble territorial des Ngicuro. Comme nous l'avons vu précédemment, le terme est issu de acurur (pl. ngacuro) qui désigne un canyon, un élément particulièrement saillant dans l'appréhension turkana de l'espace montagneux étant donné son rapport étroit à l'eau. "Les gens de l'Est et du centre » se donnent le nom de Ngimonia. Le terme provient de amoni (pl. ngamoni) qui, spécifiquement, signifie « la brousse dense », " la forêt-galerie », constituée de broussailles épineuses et de quelques arbres. Mais, le sens général de amoni est très semblable à celui que nous attribuons à « brousse » en français ou à « bush » en anglais. La plaine du Turkan est principalement recouverte de ce type de végétation (hormis les grandes zones herbeuses du Nord et les régions arides du centre).

$\mathrm{Au}$ vu de cette brève présentation, il appert que l'espace dans lequel se meuvent les Turkana est appréhendé par ceux-ci en fonction de ces deux axes fondamentaux. Nous verrons, dans l'étude des rituels, que l'axe Est-Ouest est toujours pris en compte et joue un rôle prépondérant.

\section{LA MATRICE SPATIALE DES RITUELS}

Pour compléter ma présentation de la valeur des points cardinaux too et kide, j'analyserai, dans un premier temps, la spatialisation d'une action traditionnelle turkana. Je ne reprendrai que les éléments pertinents pour mon propos, laissant de côté de nombreux aspects de la procédure. En particulier, je n'analyserai pas l'acte sacrificiel en tant que tel. Le rituel sacrificiel Akiriket me servira d'exemple. Ce sacrifice peut être exécuté pour répondre à une infortune plus ou moins grave : pour soigner 
quelqu'un (akisicumari) ou pour contrer une influence néfaste (akisicumakini). Il peut aussi être exécuté pour remercier et honorer (akipeyokin) les anciens (ngikasukou) qui, en retour, dispensent leur « bénédictions » (apiari). Il peut servir aussi une fonction apotropaïque, pour apaiser les ancêtres (akiar ngikaram, « tuer pour les ancêtres/ décharnés »). Il peut encore être exécuté sur simple décision de quelques amis. La procédure constitue, dans ce dernier cas, une " manière de table » sans objectif autre que la consommation collective d'un animal.

Comme nous pouvons le constater, une procédure identique répond donc à des situations très variées, des plus anodines aux plus périlleuses. Certains éléments changeront cependant. Les participants insisteront sur certaines actions. Le climat rituel sera modifié en accord avec l'aspect pressant ou non du problème. Les gestes des acteurs seront plus ou moins tendus. L'humeur des ngikasukou sera plus ou moins ombrageuse. Aux abords de l'espace rituel, les enfants et, plus exceptionnellement, les femmes, pourront être tolérés dans des situations moins « marquées », alors qu'ils en seront exclus dans les cas de tension extrême. Ces variations du schéma de l'action sont à rechercher dans les raisons de l'exécution du sacrifice, explicitement formulées par les initiateurs de la procédure. Il faut remarquer, cependant, que l'organisation spatiale du rituel ne se modifie jamais! Elle constitue, en ce sens, une véritable matrice, une forme minimale qui permet l'émergence de configurations relationnelles particulières $^{12}$.

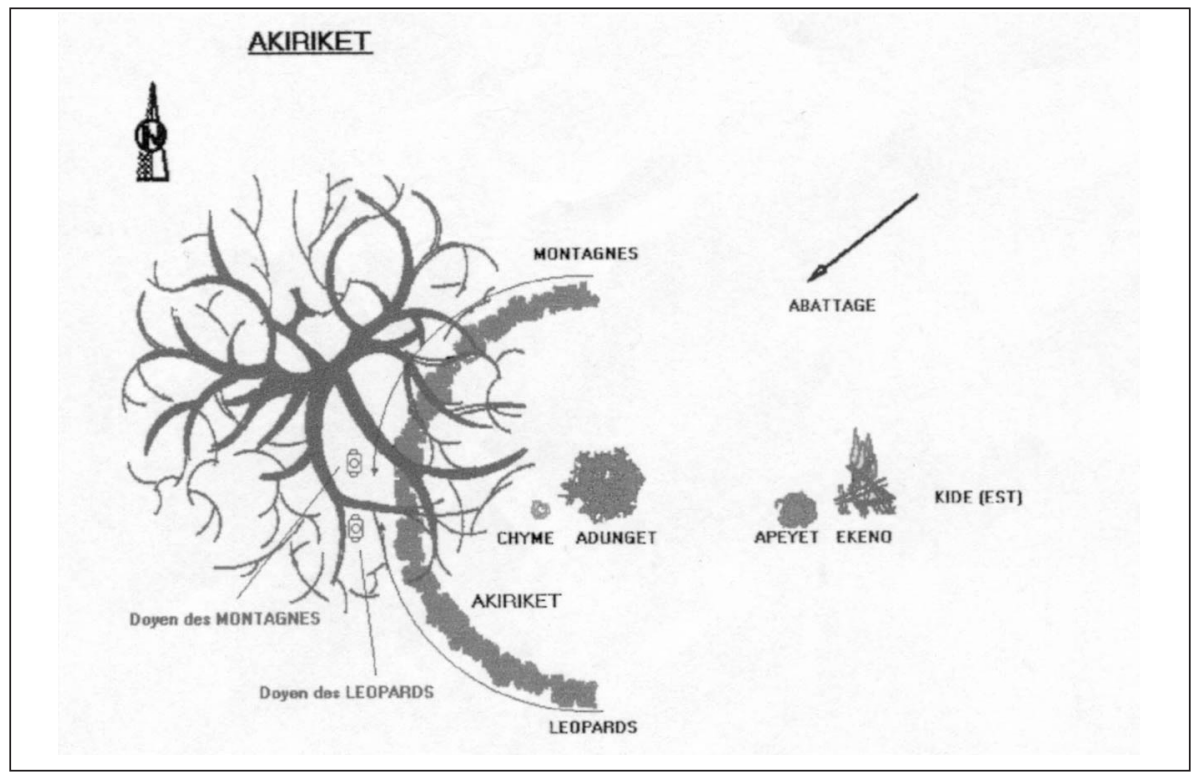

Fig. 2

L'aménagement de l'espace rituel est entrepris par des non-initiés. Un demi-cercle de branchage d'edome (Cordia sinensis), la akiriket proprement dite, est placé à même le sol, à l'ouest de l'aire du rituel, au pied de l'arbre des hommes (ekitoe à ngikiliok),

12. M. Houseman et C. Severi propose le concept de forme relationnelle qui définit particulièrement bien ce que j'ai à l'esprit (M. Houseman, C. Severi 1994). 
ouverture vers l'est ${ }^{13}$. Les « vieux initiés » (ngikasukou luapolok) s'assoiront sur son pourtour. Un amas de branchages est disposé à quelque distance à l'est de la akiriket. Il s'agit de la adunget, l'endroit de découpe, en fait le lieu où sont déposés les morceaux de viande crue avant cuisson. Plus loin encore (plusieurs dizaines de mètres) vers l'orient, se retrouve un autre petit tas de branchages d'edome, la apeyet, l'endroit où sont déposés les morceaux sortis brûlant du feu avant d'être re-transportés vers la adunget. A proximité immédiate de la apeyet, le foyer, ekeno, est le dernier élément localisé à l'extrême Est de l'aire rituelle.

Les personnes admises autour du demi-cercle de branchages sont les anciens (ngikasukou). Leur place est déterminée en fonction de l'ancienneté de leur initiation (hiérarchie des classes d'âge) et de «l'espèce » sociale (génération) à laquelle ils appartiennent. Le groupe des anciens est donc divisé en deux en accord avec leur appartenance générationnelle. Pour un observateur en position de vis-à-vis, à sa gauche est installée la génération des Léopards, à sa droite, la génération des Montagnes. Les plus jeunes des anciens de chacune des deux espèces sociales, appartenant à la classe d'âge dont l'initiation est la plus récente, occupent l'extrémité de l'arc de cercle réservée à leur génération. Les doyens des anciens des deux générations occupent le centre de l'arc de cercle. Les autres initiés admis autour de la akiriket s'ordonnent entre ces deux pôles eu égard à leur grade d'ancienneté et à leur appartenance à l'une ou l'autre des deux générations. Mais les ngikasukou ne sont pas les seuls à respecter cette démarcation entre générations. Les initiés (qui ne sont pas encore des anciens), les jeunes initiés et les jeunes hommes non initiés (proche de l'initiation) qui ne sont pas occupés à la préparation de l'espace rituel s'agglutinent à faible distance en deux groupes distincts de part et d'autre du demi-cercle, selon leur appartenance à la catégorie sociale des Montagnes ou des Léopards.

L'animal sacrificiel est généralement un animal castré. Il est soit un bovin, un caprin, un ovin ou un camélidé. Le choix du type de bétail relève d'éléments contextuels: cela peut être lié à la question de la disponibilité d'un animal, à l'importance de l'infortune à combattre, à la richesse du sacrifiant, au nombre de convives, à une révélation onirique... L'animal est sacrifié d'un coup de lance au cœur soit par un initié (asapanit) en position de « fils » (classificatoire) par rapport aux anciens soit par les anciens eux-mêmes. Le cas d'un abattage par un non-initié est plus rare mais il existe. Je ne traiterai pas de ces cas limites (infortune grave, rituels post-homicide,...).

Le découpage de la carcasse de la victime est accompli par un initié «confirmé » (qui ne s'assied pas encore autour du demi-cercle de branchages mais qui n'est plus un jeune initié). Il est aidé dans cette entreprise par des jeunes hommes non initiés. Ceux-ci transportent les morceaux de viande vers la adunget et sur ordre des ngikasukou les transfèrent vers le foyer où d'autres jeunes hommes s'occupent de la cuisson. Une maxime souvent répétée au quotidien traduit linguistiquement le rapport

13. Lorsqu'un campement s'installe dans un nouvel emplacement, les hommes se choisissent un (ou plusieurs) arbre(s) qui offre(nt) une ombre généreuse. Ces arbres sont le plus souvent localisés hors de l'espace du campement, un peu à l'écart. Ce sont les endroits où se retrouve l'ensemble de la collectivité masculine pour discuter, se reposer ou festoyer. La présence des femmes et des enfants n'est généralement pas souhaitée. Il n’y a pas de symbolique particulière attachée à ces arbres. Ils sont choisis pour le couvert qu'ils donnent et l'on en change sans états d'âme. Il est fréquent aussi que plusieurs arbres proches les un des autres servent à différentes heures de la journée parce qu'ils offrent un meilleur ombrage (l'endroit sera toujours désigné par le singulier : ekitoi a ngikiliok, l'arbre des hommes). 
que les cadets entretiennent avec leur aînés et réciproquement. Il est dit que « les jeunes rôtissent pour les anciens ». Ceux-ci, en retour, dispensent aux cadets leurs bénédictions. Ces bénédictions prennent la forme de harangues (agata). Les anciens, collectivement, enjoignent à leurs cadets de « devenir riches », « d'avoir beaucoup de bétail, de femmes, d'enfants », « de devenir vieux » et " d'être transportés par un âne» (une figure poétique imageant la très grande vieillesse) ${ }^{14}$.

Comme nous avons pu le constater, la procédure rituelle s'articule le long de l'axe kide/too. La maîtrise générale du processus revient aux ngikasukou. Mais, si nous observons dans le détail les activités des trois groupes présents sur l'aire rituelle, je peux dégager un schème logique intéressant pour mon propos. La partie Ouest de l'aire rituelle est dominée par les anciens tandis que l'Est, où se trouve le foyer, est sous le contrôle (relatif, bien-entendu) des non-initiés. Les activités entre ces deux pôles sont mixtes : les gestes précis (abattage et boucherie) sont exécutés par des initiés qui sont secondés par des non-initiés en position de servants. En adaptant à notre propos la suggestion que fait $\mathrm{M}$. Bloch de la relation entre les processus religieux et les notions de vie et de mort biologique dans son ouvrage La violence du religieux, j'affirmerai que l'action traditionnelle Akiriket est une représentation rituelle de l'existence des êtres humains dans le temps (Bloch 1997 : 14).

En quelque sorte, dans la Akiriket nous assistons donc à la dramatisation du cycle de vie masculin tel qu'il est représenté et conçu dans l'univers turkana. Les participants occupent des positions déterminées par leur statut de non-initié ou d'initié. L'ordonnancement se fait par rapport à l'axe kide/too. Un individu vieillissant s'écartera progressivement de kide pour se rapprocher de la akiriket (le demi-cercle) à l'ouest du champ rituel. Ainsi, l'enfant et le jeune adolescent sont exclus de l'aire rituelle. Leur présence est tolérée à bonne distance. L'adolescent et le jeune homme, par contre, participent à l'action. Ils occupent un espace qui leur est génériquement attribué dans l'aire rituelle (ils « rôtissent »). Progressivement, ils vont se rapprocher de la victime sacrificielle. Ils apprennent les gestes adéquats pour le traitement de la carcasse sous la direction de leur aînés. Ils sont donc habilités à participer aux rituels pour acquérir progressivement les compétences nécessaires. Les jeunes initiés et les initiés confirmés secondent les anciens et dirigent le processus pour ces derniers. Ils ont acquis le droit de sacrifier lors de leur initiation. Ils peuvent maintenant « produire » du rituel. Les ngikasukou, au crépuscule de leur vie, bénéficient de la procédure rituelle. Répartis de part et d'autre de la akiriket, par ordre d'ancienneté, ils « siègent » de plein droit. Ils consomment les chairs de l'animal et dispensent leurs bénédictions.

Le recours à l'axe de la course du soleil pour représenter l'évolution d'un homme, de ses premiers pas comme «être social» jusqu'à sa sénescence, est cohérent avec les représentations indigènes de l'univers turkana. La polarisation instituée dans le rituel fait ainsi écho aux autres polarisations rencontrées dans les représentations des membres de la société, que ces représentations soient liées à l'insertion de la société turkana dans l'écosystème aride du Nord-Ouest kenyan ou dans la configuration des ethnies présentes dans la région.

14. Comme j'ai pu le remarquer à plusieurs reprises, c'est effectivement le mode de déplacement pour les vieilles personnes qui, incapables de se mouvoir seules pour de longue marche, persistent à vouloir transhumer et refusent de se fixer aux abords d'une agglomération. 
La nécessité de limiter le sujet au cadre d'un article ne m'a pas permis de vous présenter l'ensemble des éléments qui étayent cette reconstruction logique. J'en mentionnerai encore deux brièvement. Le premier de ceux-ci nous donne un nouvel indice de la valeur qu'accordent les Turkana au concept kide. Lors de l'exécution du rituel Akiriket dans des contextes «prestigieux " (lorsque de nombreux hommes y participent, par exemple) ou dans des situations d'infortune grave, un rite dont je n'ai pas encore parlé est accompli. Les hommes, un à un, se présentent à la adunget. Là, ils s'enduisent la face antérieure du corps (le front, l'arête du nez, le poitrail, le ventre, les cuisses et les tibias) du chyme de la victime sacrificielle. Ils s'élancent alors à tour de rôle dans une course vers l'est, mimant des actes guerriers tout en poussant des cris puissants. Ils dépassent l'endroit du feu, s'immobilisent et s'exclament, face à kide, bras tendus vers le ciel : nakinai akiyar, nakinai ngibaren, ngaberu, ngide, etc, « donne-moi la vie, donne-moi du bétail, des femmes, des enfants, etc. » (ou des variantes proches de cette formulation).

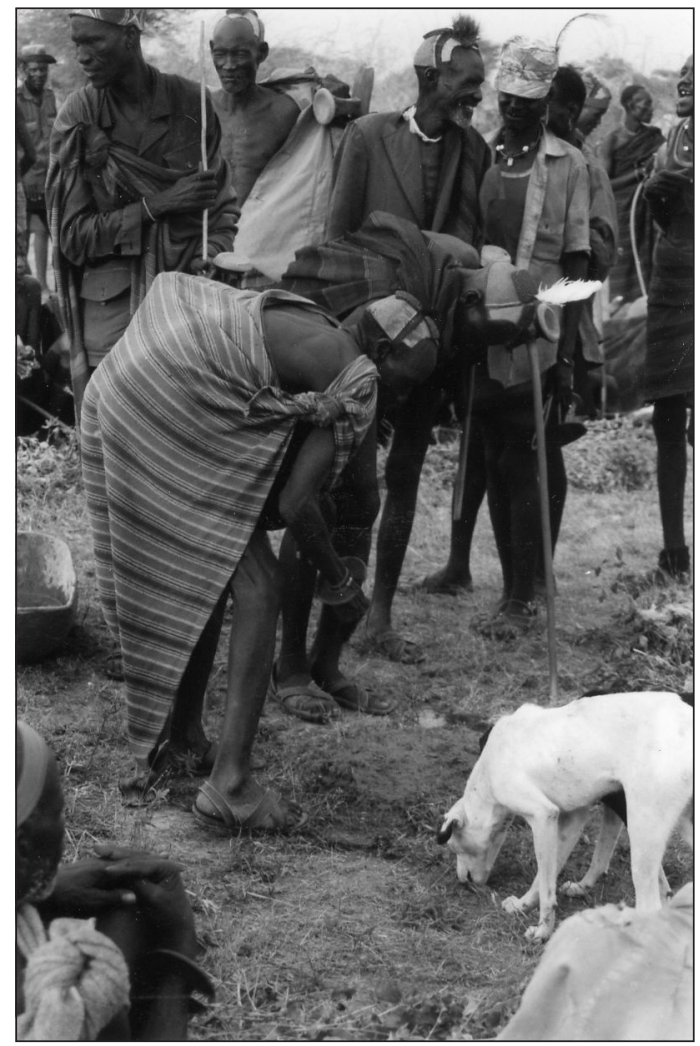

Photo 1 : Ekimomwar - Akiriket de fin de rituel

Les hommes se présentent à la adunget pour s'y enduire du chyme de la victime sacrificielle. Ils font ces onctions en regardant vers kide. (Pierre Liénard 1998 (C) Africa Museum Tervuren)

$\mathrm{Au}$ vu de ces informations, kide semble donc bien être conçu dans le rituel comme le lieu de la vitalité en opposition évidente avec l'autre point cardinal extrême de la Akiriket, too. Les rituels organisés pour la levée de deuil (ngapunyas) nous permettent 
d'appréhender la valeur de ce deuxième point, too. Lors de ces procédures, l'orientation générale de l'action est inversée par rapport à celle de la Akiriket. L'aire du rituel est placée à l'ouest de l'habitat de la famille du défunt. On y consomme la chair de la victime sacrificielle tourné vers l'ouest. Le demi-cercle de branchages est absent. La viande est déposée à même le sol. C'est en ce sens qu'il est dit que l'on «mange la terre » (akinyam ngalup). Les hommes se tiennent en groupes sans distinction entre aînés et cadets.

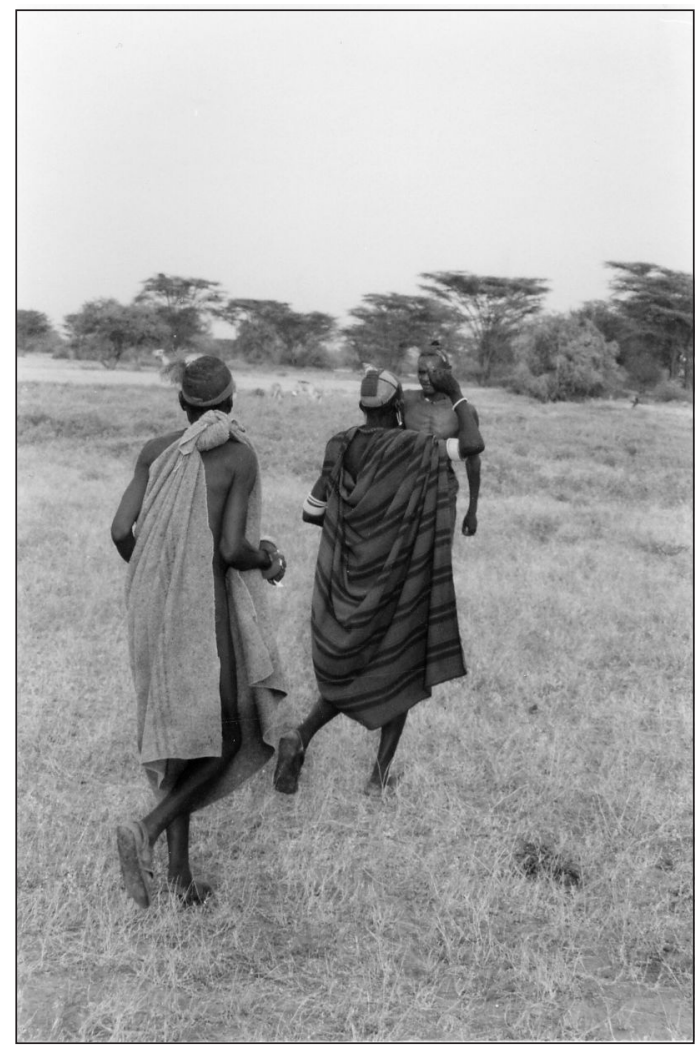

Photo 2 : Ekimomwar - Akiriket de fin de rituel

Après les onctions à la adunget, les hommes s'élancent vers kide en mimant une charge guerrière (Pierre Liénard 1998 (C) Africa Museum Tervuren)

Il semble donc que too comme kide soient conceptualisés par les membres de la population comme des pôles contraires. Je crois que nous pouvons parler d'une véritable conceptualisation même si le besoin de formaliser explicitement le rapport entre les deux concepts et leurs contenus respectifs n'est pas souvent exprimé. A l'évidence, les comportements des acteurs du rituel sont partiellement gouvernés par le potentiel inférentiel de ces deux concepts. Mais, sans savoir ce qui de la poule ou de l'œuf vient en premier, je pourrais tout aussi bien affirmer que les procédures rituelles concourent à spécifier (instaurent!) le contenu des concepts pour les participants et spectateurs de tous âges. 
Le rituel que je vais maintenant aborder présente des pôles conceptualisés de manière similaire. Too y est conçu comme un lieu mortifère et chaotique, kide comme un lieu de vie et d'ordre. Dans un esprit de concision, je n'expliciterai pas l'ensemble des références que nécessiterait une compréhension approfondie du rituel. Je n'aborderai donc pas l'ensemble des identifications entre sacrificateurs, sacrifiants et sacrifiés ni le traitement spécifique du corps des victimes sacrificielles.

\section{L'EKIMOMWAR $^{15}$}

Nous sommes au mois de janvier 1998. Apa Mulele, un devin de la section territoriale des Ngilukumong, lors d'un de ses séjours en ville, a une révélation onirique. Une infortune grave menace sa section territoriale. La spécification du type de menace reste assez vague. Il semble qu'une épidémie risque de se propager. Une chose est certaine cependant: l'époque est néfaste. La population, qui avait entamé un déménagement vers de meilleurs pâturages, l'interrompt et revient sur ses pas, à proximité de l'espace du camp récemment quitté. C'est le début de l'Ekimomwar, un rituel qui durera quatre nuits et quatre jours.

Le terme utilisé pour dénommer le rituel désigne aussi une danse collective. Ce type de danse est pratiqué lors des mariages " pour honorer la belle-famille », avant toute autre danse « pour que celle-ci soit bonne », au sortir de la saison sèche, après les premières précipitations, « pour le retour des pluies » ou lors d'une situation dramatique ponctuelle pour combattre l'infortune qui menace l'ensemble de la population. Nous sommes dans ce dernier cas de figure.

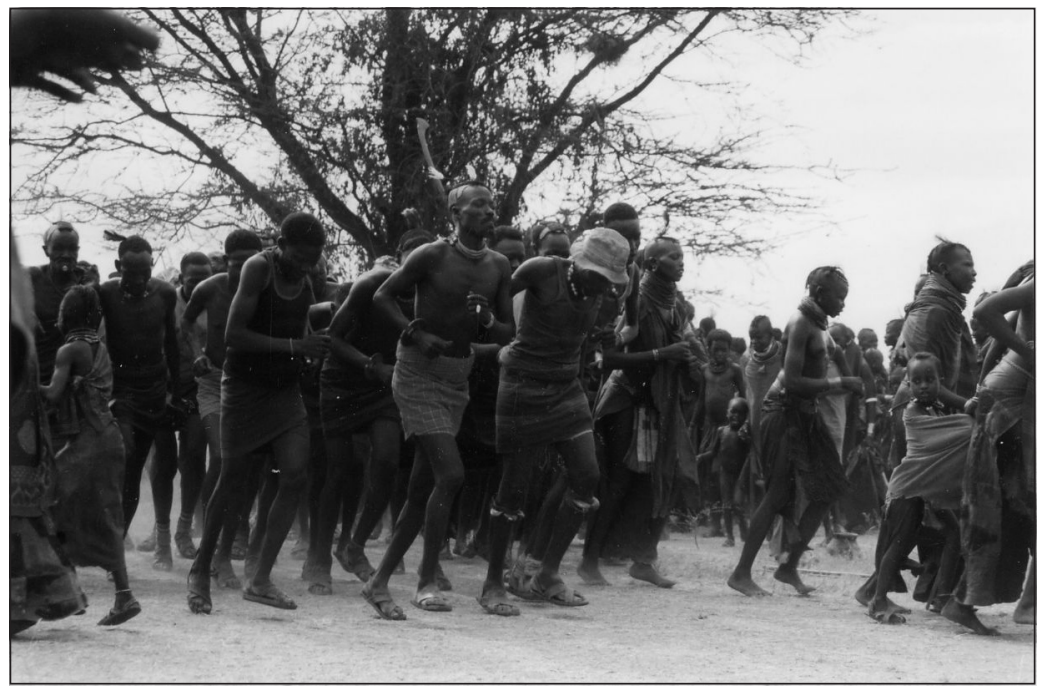

Photo 3 : Ekimomwar-aire de danse

Scène de danse. Le devin (en chapeau) conduit les danseurs. (Pierre Liénard 1998 (C) Africa Museum Tervuren)

15. Le lecteur trouvera, entre autres choses, une analyse détaillée de ce rituel dans la thèse de doctorat de l'auteur (Le comportement rituel : communication, cognition, et action. Génération, âge, filiation et territoire : contribution à l'ethnographie de deux populations du Cercle Karimojong (les Turkana $d u$ Kenya et les Nyangatom d'Ethiopie)). 
Le rituel analysé est exceptionnel à plus d'un titre. De la manière de tuer les animaux sacrificiels (découpé vivant au niveau du sternum et égorgé au moyen d'une lance) au traitement auquel sont soumises les diverses composantes sociales (l'agnation et la section territoriale), de nombreux traits diffèrent du modèle sacrificiel de la Akiriket. Un élément reste cependant identique : le recours à l'axe solaire pour orienter l'action. Le rituel de l'Ekimomwar organise en fait un déplacement d'ouest en est : après une première phase préparatoire longue et épuisante à l'Ouest, les participants se dirigent vers l'extrême Est de l'aire rituelle où se termine le rituel.

Le soir de la réinstallation à proximité de l'ancien camp, le devin rejette la maladie/infortune (edeke) en direction des montagnes de l'Ouest. Il entame ensuite une répétition générale des danses qui occuperont la majorité des séquences rituelles préparatoires aux sacrifices. Les jeunes apprennent aussi les chants de l'Ekimomwar.

Le lendemain matin, deux pots de terre cuite sont déposés au cœur de ce qui sera désormais l'aire de danse (akuor) pour toute la durée du rituel. Ces pots sont appelés les « puits de la akuor $»^{16}$.

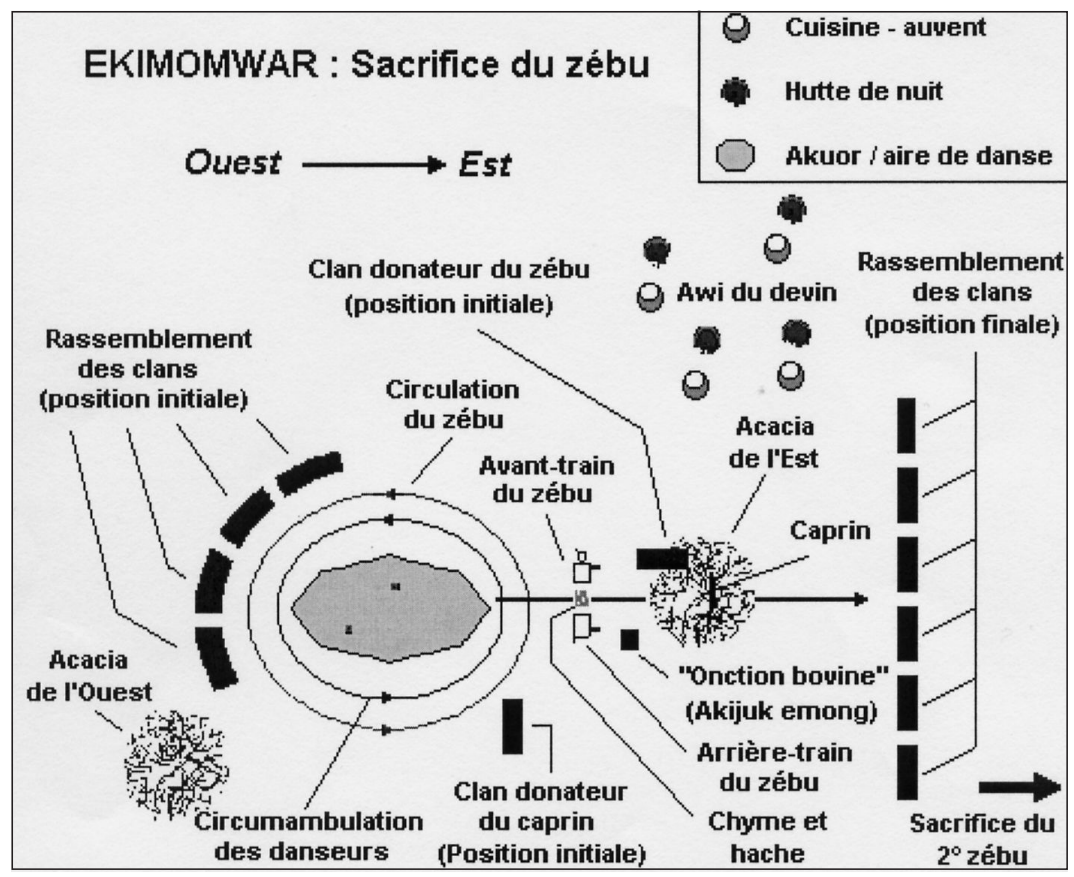

Fig. 3

Pendant cette journée et celle du lendemain, la population converge de l'ensemble du territoire de la section. Ces deux premiers jours servent manifestement à l'amorçage du rituel. On danse et on chante sans s'arrêter sauf pour les activités de la traite et, tard dans la nuit, pour se reposer brièvement. Il est impératif pour le succès de l'entreprise que tous s'efforcent de participer sans interruption. Le devin et quelques vieux initiés veillent au respect de la règle. Ils tournent autour des danseurs et leur assènent des coups de badine.

16. Cfr. ci-dessous la présentation d'un des chants de l'Ekimomwar. 


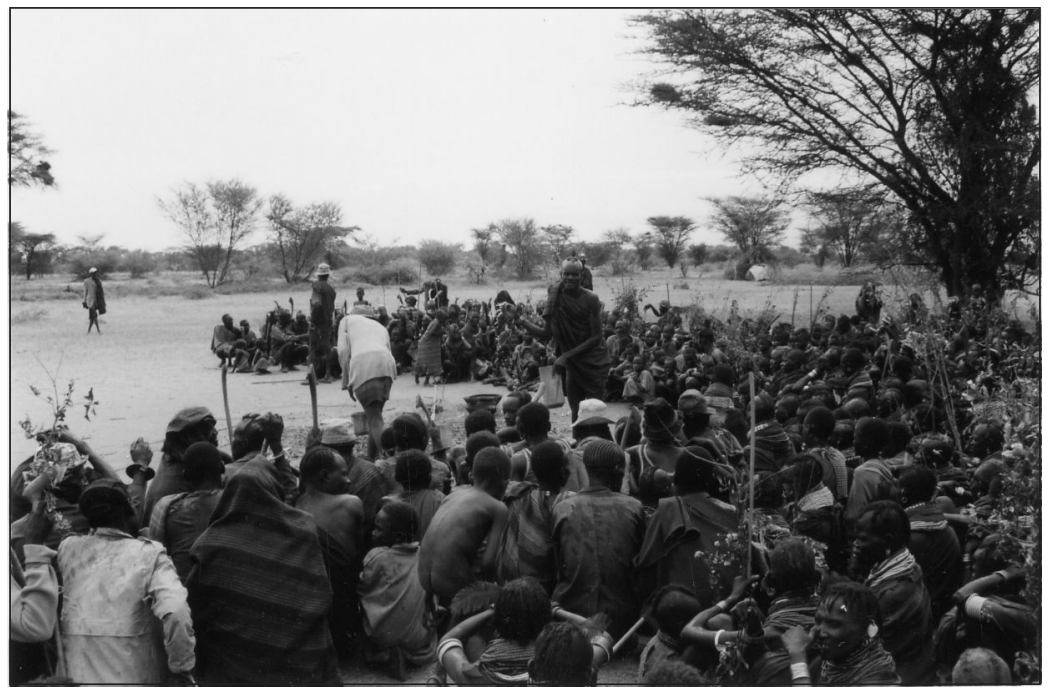

Photo 4 : Ekimomwar - aire de danse, aspersions.

Le devin et ses assistants aspergent les danseurs rassemblés en demi-cercle à l'ouest de l'aire rituelle (Pierre Liénard 1998 (c) Africa Museum Tervuren)

A chaque fois qu'une interruption se produit (pour des impératifs pastoraux ou pour terminer une session de danse) les participants doivent subir tour à tour des aspersions libérales d'eau et des « flagellations ». Pour ce faire, les danseurs s'assemblent à l'Ouest de l'aire du rituel, en forme de demi-cercle, regardant vers kide. Ils sont aspergés par le devin et ses aides avant de traverser l'aire de danse et de passer, un à un, une catégorie sociale après l'autre (femmes, enfants, initiés, jeunes filles et non-initiés) entre les deux poteries. C'est à leur passage qu'ils se font flageller. La sortie des danseurs se fera toujours par kide/l'Est.

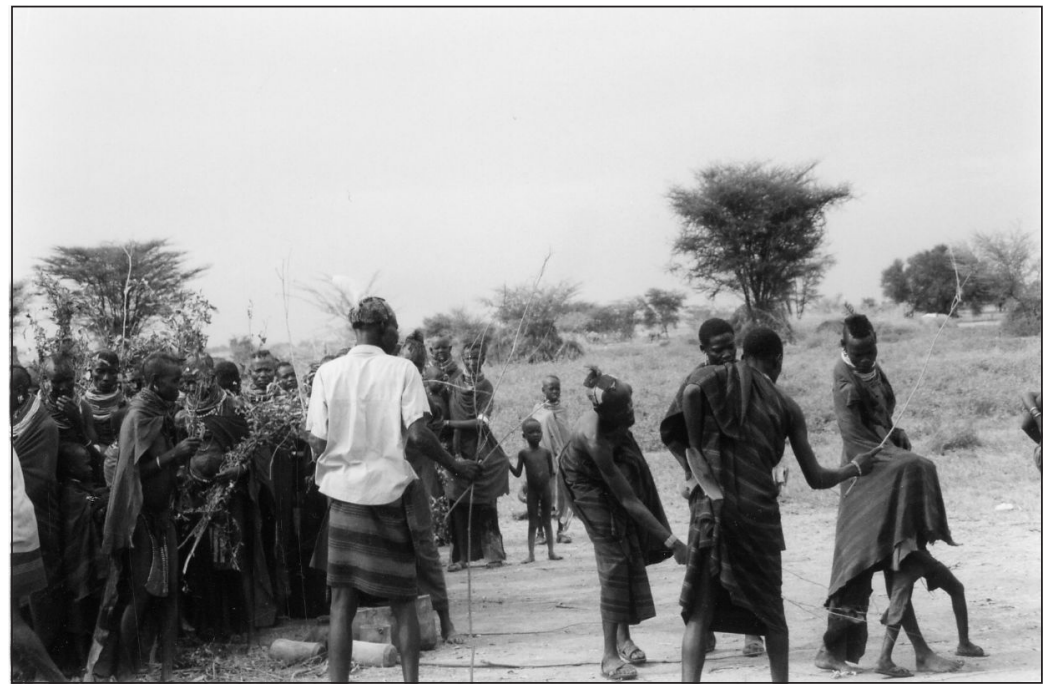

Photo 5 : Ekimomwar - aire de danse, flagellation au passage entre les deux puits de la akuor. Les assistants du devin s'efforcent de flageller une jeune fille qui se cache sous le tablier d'une femme. (Pierre Liénard 1998 (C) Africa Museum Tervuren) 
Selon mes informateurs, les danses et les chants qui les accompagnent servent à « ouvrir l'aire de danse » (angar akuor), un espace chorégraphique et sonore, qui, par la suite, ne sera plus oublié. Ce lieu, ainsi physiquement délimité par les corps et par les voix, devient un lieu de mémoire sur lequel on pourra faire retour le cas échéant (lors d'une autre épidémie, par exemple) ${ }^{17}$.

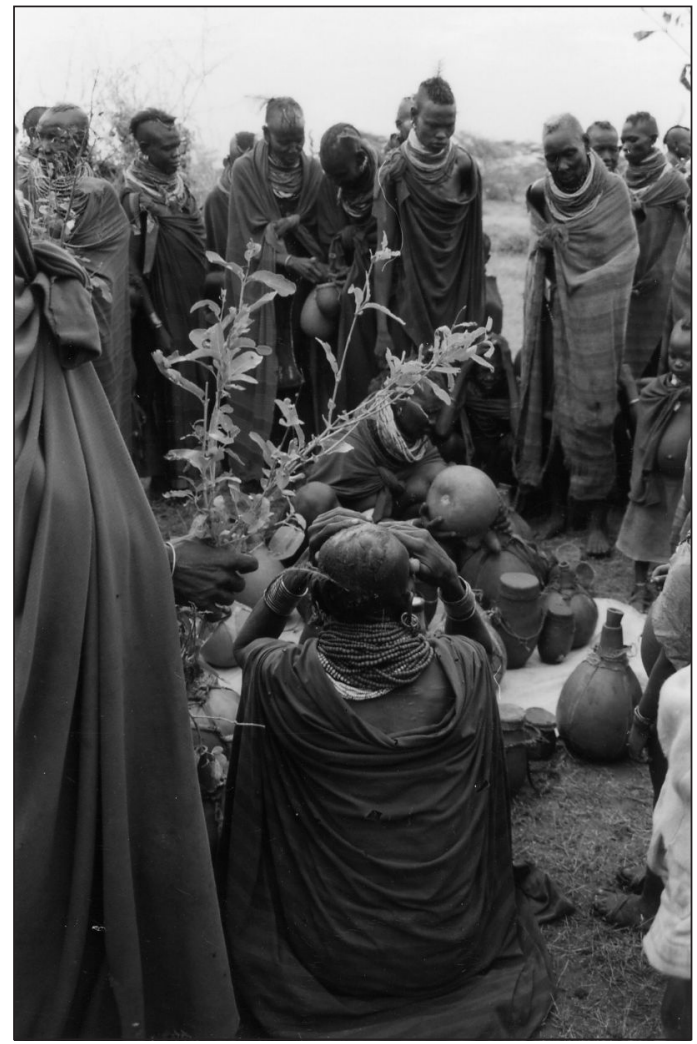

Photo 6 : Ekimomwar - «ferme » du devin, traitement du lait.

Les « mères » apportent un conteneur de crème et une calebasse de lait frais. La sœur du devin transvase dans une grande calebasse le lait apporté par les femmes d'un même clan. Les récipients resteront au soleil durant toute la journée.

(Pierre Liénard 1998 C Africa Museum Tervuren)

17. Chaque section territoriale possède plusieurs akuor/aires de danses où peuvent être exécutés de grands rituels propitiatoires (amuronot). L'aire où se tient le rituel décrit ici est « ouverte » pour la première fois. L'ensemble de ces lieux " (sur-)marqués » concourent à construire une véritable géographie de lieux-dits. La dénomination de ces derniers fait souvent référence à un détail de l'environnement: la forme particulière d'une roche visible dans le lointain, une rivière, un champ de pâturages clairement délimité par des particularités du relief... Certains lieux-dits portent des noms qui font référence à des événements lointains: par exemple dans la région de Lorugumu (section territoriale Ngikamatak), le «Champs des os/squelettes » qui renvoie à un événement guerrier. D'autres, encore, font référence à des entités surnaturelles: dans le centre-Sud du Turkan (région proche de Kailongol) se trouve l'ekite$l a$ de Marakalo, le «plateau » de Marakalo, un esprit (ekipe). L'espace turkana est ainsi divisé en une multitude de points de référence « absolus » que transcendent les axes d'orientation généraux que sont kuju/kwap et too/kide. 
Le matin du troisième jour, le traitement rituel du lait et de ses dérivés commence. Des récipients contenant du lait frais et de la crème sont déposés sur une peau de zébu au cœur de la $a w i / «$ ferme » du devin. Ils y resteront en plein soleil toute la journée. Les participants retournent ensuite vers l'aire de danse pour y danser à nouveau. En fin d'après-midi, les membres de l'assemblée forment une grande chaîne qui va ceinturer une aire de plus en plus grande autour de la akuor.

A l'ouest de l'aire rituelle, quelques jeunes ont dégagé des broussailles qui l'entourent un acacia tortilis. La chaîne des participants se met lentement en marche (sens anti-horloger). La procession passe à quatre reprises dans la fourche naturelle constituée par le tronc bifide de l'arbre. Pour les deux derniers passages, la masse des danseurs est divisée en groupes sociaux différenciés : les hommes viennent en premier, ensuite les jeunes filles, les mères et les enfants et finalement les jeunes hommes non-initiés. Après cette séquence rituelle, les participants se rassemblent en demi-cercle (ouverture vers l'est) à l'Ouest de l'aire rituelle, face aux poteries. Le devin et ses assistants aspergent la foule avant le passage entre les puits de la akuor et les flagellations qui accompagnent toujours cette séquence.

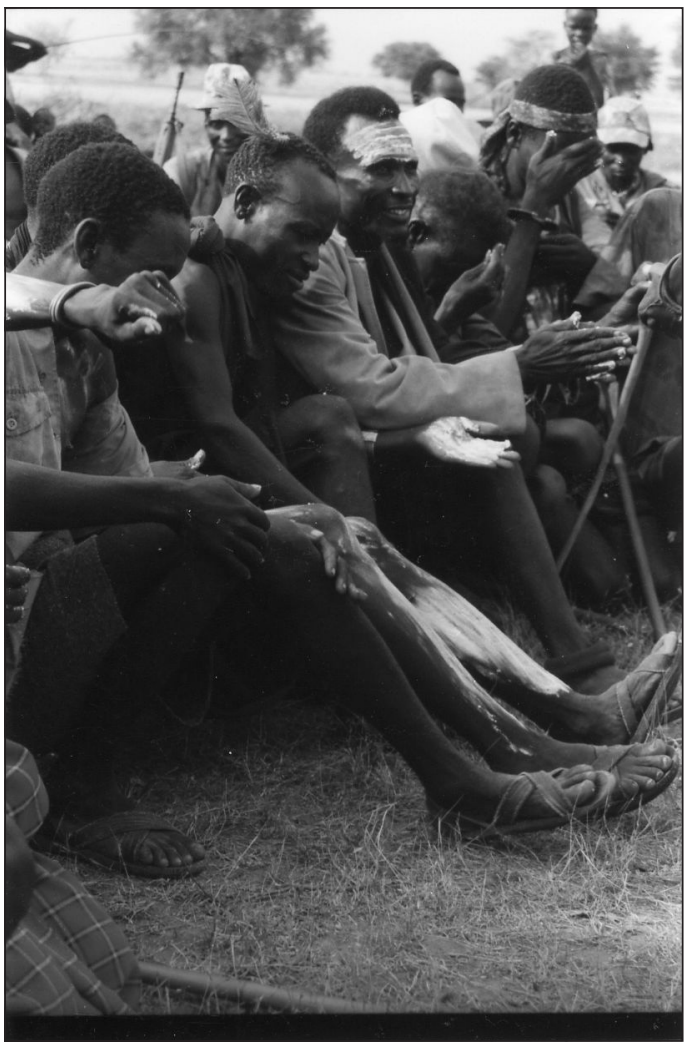

Photo 7 : Ekimomwar - «ferme» du devin, onctions de crème. Des jeunes hommes non initiés s'enduisent de crème en regardant vers kide. (Pierre Liénard 1998 (C) Africa Museum Tervuren) 
La communauté des danseurs se retrouve ensuite à la awi du devin. Tous se badigeonnent le front, la poitrine et les jambes de crème et d'huile animale. Il est impératif d'accomplir ces onctions en regardant vers l'est. Le lait est ensuite consommé (par les aînés des hommes exclusivement). Un interdit majeur conditionne le partage du lait : personne ne peut boire le lait de son clan. La journée se termine sur cet épisode rituel.

Dès l'aube du quatrième et dernier jour, les danses de l'Ekimomwar reprennent. Elles se prolongent jusqu'à l'arrivée de l'animal sacrificiel, un bœuf de robe blanche. Le bovin, suivi de la famille du propriétaire, des anciens et des hommes, tourne par trois fois autour des danseurs. La ronde est ensuite interrompue et l'assistance s'assied à l'ouest des poteries.

L'heure suivante est passée à la préparation de l'aire du dernier rite. A l'est du champ rituel, un autre acacia est dégagé de ses broussailles et aménagé en un deuxième «seuil» pendant que l'assemblée se divise par groupes de clans. L'animal est amené à l'acacia de l'Est pour y être plaqué au sol et solidement attaché. Un par un, les membres du clan donateur viennent se frotter (akijuk, " oindre » ou « enduire ») au chanfrein du zébu.

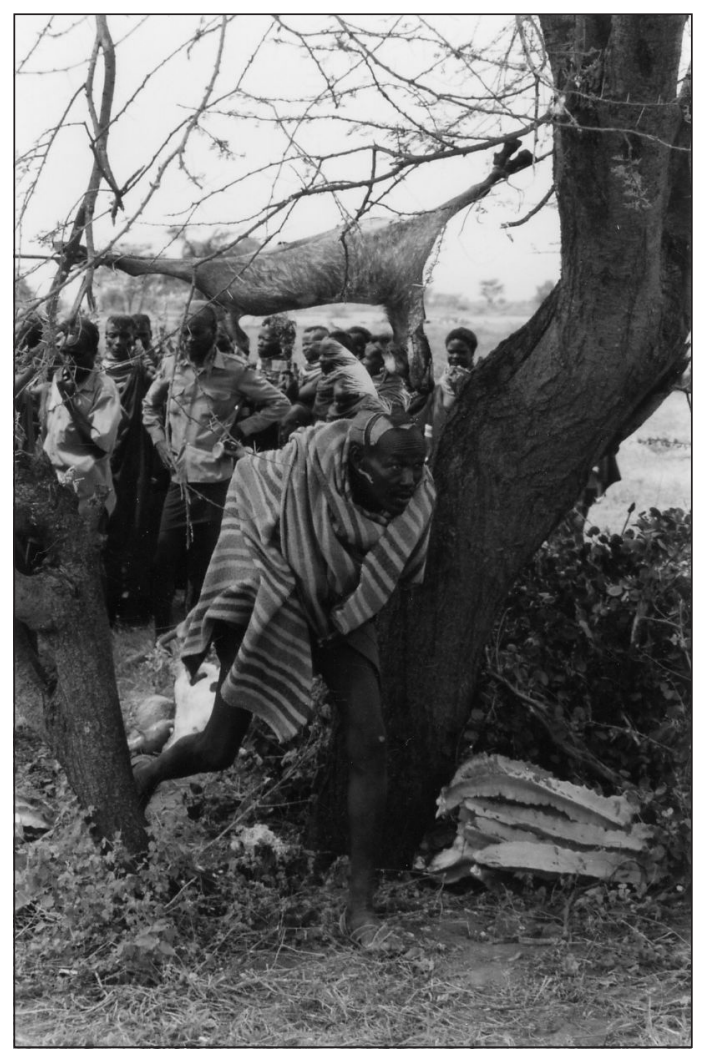

Photo 8 : Ekimomwar - scène du dernier rite.

Le sacrificateur du caprin passe en second, entre les morceaux du corps du zébu et sous le jeune bouc, après le sacrificateur du premier animal.

(Pierre Liénard 1998 (C) Africa Museum Tervuren) 
L'animal est ensuite découpé vivant au niveau du sternum par un initié du clan donateur ${ }^{18}$. Une fois l'ouverture assez grande, l'estomac est extrait de la panse et ouvert pour en récolter le contenu ${ }^{19}$. La victime est finalement mise à mort de deux coups de hache qui sectionnent la colonne vertébrale de l'animal. Les deux parties du corps de la bête sont placées, en amont de l'acacia de l'Est, de part et d'autre de la voie menant à la porte/seuil. Entre les deux morceaux du corps de la victime, le devin répand du chyme. Il y enfonce la hache utilisée pour la mise à mort. Un deuxième animal sacrificiel, un jeune bouc, est apporté par un membre d'un deuxième clan. Le caprin est suspendu aux branches de l'acacia de l'Est, transversalement par rapport à l'axe kide/too. Le sacrificateur (issu du clan donateur du caprin) lui donne la mort en lui sectionnant les carotides avec la lance qui a servi à découper le zébu. La structuration de l'aire du dernier rite est ainsi terminée.

En ordre précis, les clans vont traverser la porte/seuil, les deux clans donateurs en tête. Au sein de chaque clan, les anciens et les initiés passent en premiers. Les jeunes filles, les femmes et les enfants suivent. Les derniers à passer sont les jeunes noninitiés. Lors du passage, il est impératif que tous posent le pied sur la hache enfouie dans le chyme avant de poursuivre leur chemin et de passer sous la chèvre égorgée suspendue dans l'acacia. Les participants se dirigent ensuite vers kide, les bras levés vers le ciel.

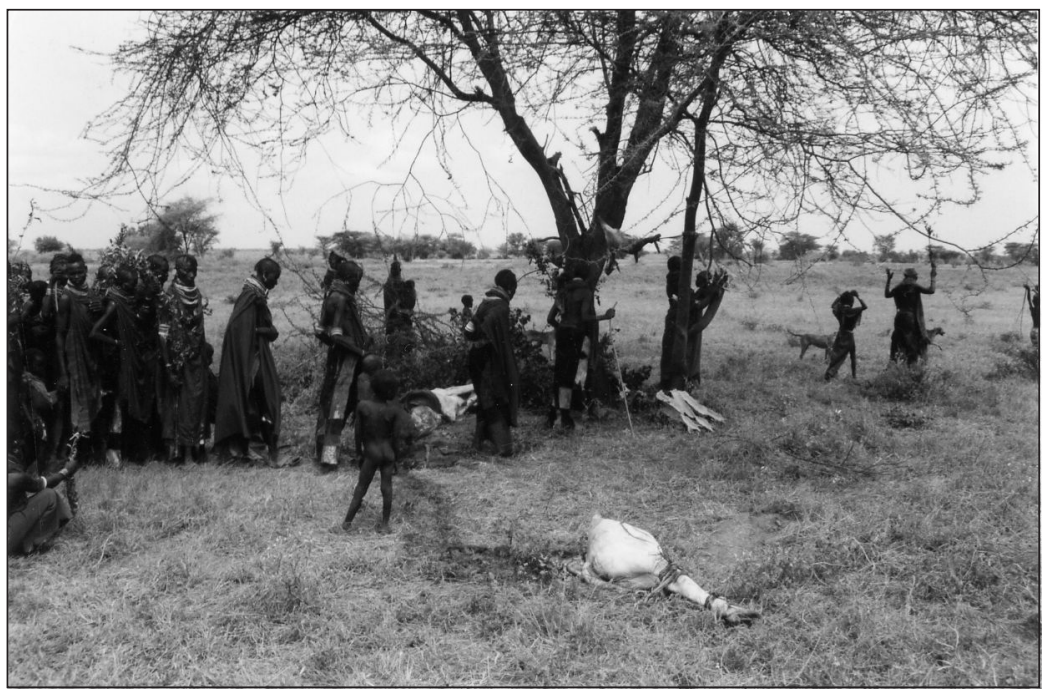

Photo 9 : Ekimomwar - scène du dernier rite.

Les femmes et les enfants d'un clan passent la "porte/seuil » de l'Est. Les mères progressent ensuite vers kide, les bras levés vers le ciel.

(Pierre Liénard 1998 C Africa Museum Tervuren)

18. Dans les rituels ariwo/d'évitement de l'infortune, lorsqu'un sacrifice est requis, c'est cette forme d'abattage (corps découpé en deux perpendiculairement à la colonne) qui prévaut.

19. Cette prescription rituelle de l'ouverture de la panse de l'animal encore vivant pour y prélever du chyme (apuor, « ouvrir en découpant ») est couramment suivie dans des situations jugées dangereuses. 
Lorsque tous les clans sont passés, ils retournent dans le même ordre à la akuor pour y être aspergés une dernière fois (clan après clan) avant de regagner définitivement l'Est. Les doyens de chaque clan se lancent alors dans les harangues (agata) de fin de rituel. Après un moment, le devin conclut par l'énonciation des interdits qu'il faudra respecter pour un temps après l'Ekimomwar. Les derniers mots du devin prononcés, dans un mouvement général, les participants se ruent en direction de kide. Levant les bras vers le ciel, ils s’écrient : Akuj, kingarakinai sua! (« Ciel, aidenous »). L'Ekimomwar est suivi d'une Akiriket, un festin collectif, à laquelle tous les hommes participent.

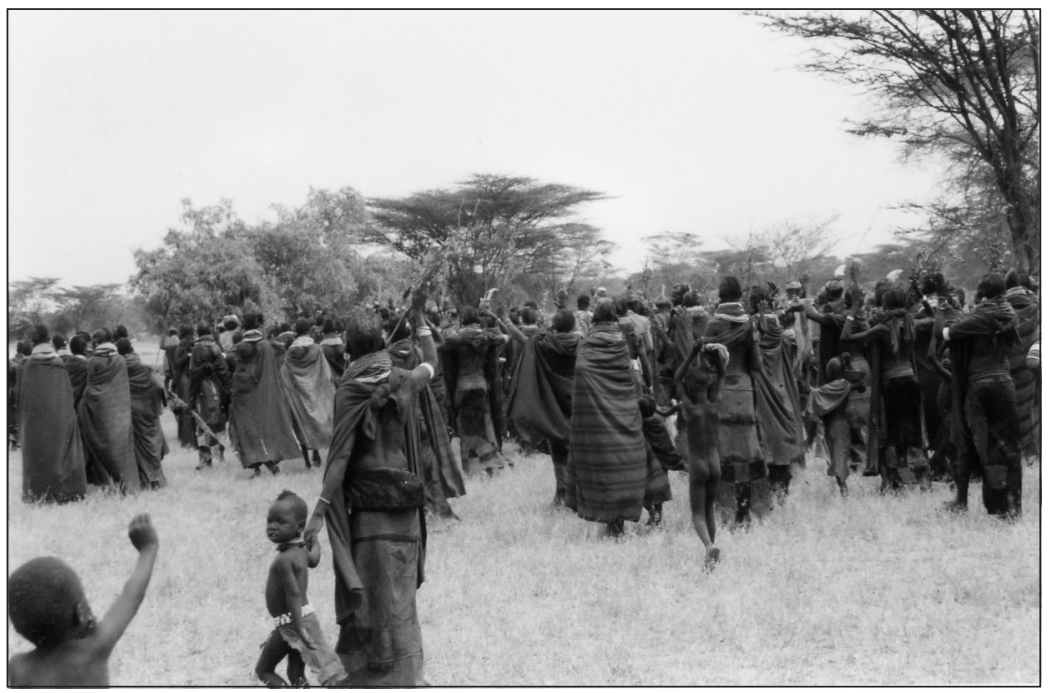

Photo 10 : Ekimomwar-scène finale

Les participants se ruent vers kide, levant les bras au ciel. (Pierre Liénard 1998 (C) Africa Museum Tervuren)

\section{L'EKIMOMWAR, EPIPHÉNOMĖNE D'UN SCHĖME LOGIQUE}

L'Ekimomwar est le lieu d'une mise en forme de certaines valeurs fondamentales de la société turkana par le traitement simultané du territoire et de l'agnation. Un espace va être sur-marqué de signes indiciels (les " puits », les « seuils »,...) pour lui donner sa qualité d'espace générique. Cette géographie n'est pas uniquement montrée ; elle est aussi évoquée verbalement. Les prières et certains chants, de fait, participent à l'instauration des valeurs antinomiques qui sous-tendent les représentations turkana de l'univers. Nous avons vu que, le soir de la répétition générale, le devin, rejetait verbalement la maladie vers l'ailleurs montagneux i.e. l'Ouest (kape tolot, kape ama, "va-t-en, va par là »). De même, un des chants répétés inlassablement durant les circumambulations autour de la akuor rappelle, de manière suggestive, la bipolarité de l'univers turkana. 
Exemple :

Devin

Chœur

Le feu a consumé les doliques des Dodos (1)

il a consumé!

Le feu a consumé les doliques des Kuman

il a consumé!

Ils ont « quitté » la montagne...(2)

Tournez autour du puit (4)

la montagne

« Nakapel »(3)

ngaiyeiya yelo (5)

1. A la fin de la saison sèche, les populations limitrophes des franges montagneuses de l'Ouganda préparent les champs pour les plantations de début de saison des pluies. Le feu est mis aux broussailles desséchées. A cette période de l'année, la montagne s'embrase et la nuit elle scintille de dizaines de foyers qui brûleront parfois pendant plusieurs jours.

2. Cette phrase est, par excellence, évocatoire. Différentes acceptions sont possibles. Ce peut être une référence à la migration de début de saison des pluies quand les pâturages de plaines reverdissent. Cette phrase peut aussi renvoyer à l'ethnogenèse turkana et à la « descente originelle » des contreforts ougandais pour s'installer dans le Turkana. Le récit de cette genèse le plus généralement raconté est celui de Nayece et du taureau gris ${ }^{20}$. Huit jeunes guerriers, venant de chez les Jie recherchent un taureau qui s'est égaré. Ils descendent les contre-forts montagneux qui marquent la frontière entre l'Ouganda et le Kenya. Ils aboutissent aux sources de la Tarash, une grande rivière du Nord du Turkan. Là, ils rencontrent Nayece, une vieille femme, elle aussi originaire du pays Jie. Le taureau vit avec elle. La région est attrayante et riche de pâturages. Les jeunes hommes rentrent dans leur pays. Ils réussissent à convaincre d'autres personnes de les suivre avec leurs troupeaux et de s'installer dans la région qui s'appelle depuis ce jour la Montagne de Nayece (Moru a Nayece). Ils devinrent les Turkana.

3. Nom propre d'une montagne du pays Jie que l'on voit au loin dans le pays Ngilukumong.

4. Les danseurs tournent autour des poteries. Les poteries en tant qu'indices permettent aux spectateurs d'avoir ce que J. Pelletier décrit comme une « expérience récognitionnelle de certaines propriétés visuelles [le contenant, l'eau contenue] de la source causale de cet indice [l'expérience, du concepteur de l'indice, des puits creusés dans les lits de rivière] » (Pelletier 2000 : 184). Du fait de cette forte iconicité de l'indice matériel, la relation causale qu'il entretient avec sa source est « transparente » : les poteries sont donc les puits de la akuor pour l'ensemble des participants.

5. Mouvement musical intraduisible.

Ce chant évoque, plus spécifiquement encore, le cheminement (Ouest/Est) que devront accomplir les danseurs dans le rituel. Mais ce déplacement physique, effectué dans le rituel, évoque, à son tour, un tout autre cheminement. J'avance l'hypothèse que, dans l'Ekimomwar, sont représentés rituellement divers processus physiologi-

20. Une version détaillée de cette histoire se trouve dans l'ouvrage de John Lamphear (1976: 91). 
ques dont celui de la guérison, tel qu'il est conçu et désiré par les acteurs sociaux ${ }^{21}$. L'assignation d'une valeur négative à un lieu de départ permet la dramatisation de ce dépassement de la maladie. Dans la logique rituelle, les danseurs sortent progressivement, de manière « réaliste » (c.a.d. par l'extériorisation du processus), de leur état morbide par un éloignement physique du pôle négatif. Plusieurs opérateurs participent à l'instauration, dans la temporalité rituelle, de cette réalité désirée : les poteries/ puits, les substances « rafraîchissantes » telles le chyme et l'eau, le lait/instrument de re-clanification, les deux acacias/seuils (l'un à l'Ouest, l'autre à l'Est), la lance, la hache, le bœuf et le bouc. Les acteurs du rituel subissent donc de manière réaliste une série de transformations qui les mènent d'une situation initiale qualifiée négativement par le devin (l'état morbide et chaotique proclamé) à une situation finale irréversible et positive (l'état de vitalité et d'ordre désiré), en passant par des paliers successifs (la phase dilatoire des danses introductives et la progression d'ouest en est en passant au cœur de l'espace sacrificiel).

\section{LE RITUEL, UNE ACTION TRADITIONNELLE}

Il est légitime de se demander pourquoi une entreprise de redéfinition de certaines composantes de la société (les groupes agnatiques, les univers féminin et masculin) peut être conçue comme efficace dans un processus rituel d'évitement de l'infortune. L'Ekimomwar est une action traditionnelle. Selon la définition qu'en donne Pascal Boyer, l'action traditionnelle est un événement répété en ce sens qu'il est reproduit en référence à des exécutions antérieures (Boyer 1990 : 2). Nous pouvons nous demander ce qui est véritablement répété. Est-ce l'ensemble des représentations et rationalisations justifiant le processus rituel? Je ne le crois pas. Comme le déclare Boyer : le fait que l'action soit traditionnelle n'implique pas qu'elle véhicule les mêmes représentations et ait les mêmes effets pour les générations successives qui l'exécutent (Boyer 1990 : 9).

Comme pour toute action conventionnelle, le fait de savoir que l'exécution d'une action rituelle a été jugée efficace par des prédécesseurs semble suffisant pour justifier sa répétition. Ce sont donc manifestement les «propriétés formelles » qui sont conçues comme efficaces dans un contexte similaire (jugé comme tel par les acteurs du rituel) aux conditions antérieures d'exécution. C'est la situation que nous rencontrons dans le cas présent. Je n'ai pu obtenir que peu de rationalisations sur les raisons d'être du rituel et sur l'efficacité que peut avoir la re-classification de la société pour le dépassement de l'infortune. Une chose est certaine cependant. Le rituel est etal a Ngiturkana, « une règle des Turkana » et il est hérité des deux premières générations (Montagnes et Léopards) qui sont dites avoir « démarré/choisi le pays ».

21. Les interprétations mettent en évidence indirectement cette conceptualisation particulière du processus de guérison. Lorsque je demandais pourquoi le devin et ses assistants frappaient les danseurs à leur sortie de l'espace rituel, il m'était répondu que c'était pour " chasser », du corps, la maladie et pour qu'elle « reste » en arrière lorsque les participants traversent l'aire rituelle d'ouest en est. Ces rationalisations facultatives (elles ne sont pas nécessaires au bon déroulement de l'action rituelle ni à son efficacité sur les états mentaux des agents) rendent manifeste une configuration sémantique particulière: le corps humain est appréhendé comme un contenant qui serait « pénétré » par la maladie. 


\section{L'INDUCTION D'UNE VISION DU MONDE}

Dans les actions rituelles sont manifestement renforcés des modèles comportementaux et un rapport spécifique à l'espace par l'attribution de valeurs positives ou négatives aux « points cardinaux ». Elle permet aux acteurs d'inférer des propositions sur le monde à partir de la présentation, de la spatialisation et de la manipulation d'indices matériels (les opérateurs de la transformation, les pôles). Le résultat de ces inférences (les propositions sur le monde qu'élabore un individu) est donc fondamentalement issu d'une expérience idiosyncrasique du monde. Toutefois, j'ai pu constater qu'il existait une forte convergence des interprétations et rationalisations (souvent minimales) présentées par les membres de la population turkana à propos des actions rituelles, de leurs constituants, de leurs objectifs et de leurs effets dans le monde ${ }^{22}$. C'est d'autant plus étonnant qu'il n'y a pas de passation d'une doxa unifiée des aînés vers les cadets ni de quelconques spécialistes vers des « laïques $»^{23}$.

Comme je l'ai soutenu auparavant, l'explication de cette convergence ne peut être recherchée dans une sorte de discours que le rituel «tiendrait» sur le monde. Il est probablement plus justifié de rechercher les causes de la convergence dans ce que J. R. Searle appelle l'arrière-plan (Searle 1995). Selon cet auteur, « les états intentionnels ne fonctionnent que relativement à un ensemble de capacités d'arrière-plan qui ne consistent pas elles-mêmes en phénomènes intentionnels » (Searle 1995 : 169). Il définit ainsi l'arrière-plan « comme l'ensemble des capacités non-intentionnelles ou pré-intentionnelles qui permettent aux états intentionnels de fonctionner » (Searle 1995 : 169).

22. On sacrifie pour contrer des influences négatives: celles des ancêtres (ngikaram) qui se manifestent entre autre dans le rêve « lorsqu'ils sont oubliés » (i.e. on n'a pas sacrifié en leur honneur), celles des amis jaloux qui sont à la source de nombreux désordres lorsqu'ils sont, eux aussi, oubliés (i.e. on a dérogé à l'impératif de sacrifier régulièrement pour ses aînés ou pour ses pairs). On sacrifie de retour de raid après avoir tué un ennemi (pour éviter le retour de l'infortune). On sacrifie pour affaiblir l'ennemi (ce qui donne lieu à l'exécution d'un nouveau type de rituel ariwo/d'évitement). Etc. Selon mes informateurs, le non-respect des procédures rituelles est toujours sanctionné. Les symptômes des sanctions sont des plus divers (gonflements, mauvaises circulations des fluides corporels, problèmes articulaires, morts brutales, surexposition à l'ennemi...). Généralement, tout trouble potentiel qu'un agent risque de connaître peut être interprété comme le symptôme de l'implication d'une agence (surnaturelle ou non) ou comme la sanction d'une infraction commise. Les formulations des risques encourus mettent en évidence un certain nombre de représentations partagées dans lesquelles bien-être physique individuel, respect de la règle et ordre social s'interpénètrent constamment. Par contre, il est beaucoup plus difficile d'obtenir des interprétations élaborées et logiques expliquant, par exemple, comment un geste rituel spécifique agit sur la cause des symptômes de la maladie ou de l'infortune. La réponse la plus courante est le renvoi à l'efficacité des exécutions antérieures (« les anciennes générations l'ont toujours fait »).

23. Je dois ici mettre un bémol à cette affirmation. Le devin propose des interprétations de situations. Il est donc bien un spécialiste. Lorsque j'affirme qu'il n'y a pas de transmission d'une doxa, je mets en évidence une caractéristique importante de la transmission de l'information entre les générations dans l'univers culturel turkana. Il n'existe pas de corps de doctrine rigide et clairement défini dans lequel serait mis en relation des comportements rituels spécifiques et leurs interprétation canonique (en quoi et comment ce que l'on fait agit sur la cause). Il existe bien par contre un ensemble de définitions floues des situations qui imposent d'exécuter différentes procédures rituelles. Les différentes techniques de divination (par les sandales, par la « lecture » des entrailles d'une victime sacrificielle,...) permettent de sélectionner une interprétation de la situation d'infortune au sein d'un ensemble limité de définitions potentielles (prédéterminées). 
Dans notre cas, ces capacités seraient, par exemple, les savoirs pratiques qu'il est nécessaire de posséder pour exécuter l'action rituelle : les comportements adéquats dans le rituel ou entre les membres de catégories sociales distinctes, les techniques de mise à mort, de boucherie, les gestes à accomplir pour configurer l'aire rituelle, les règles procédurales de l'action,... Ce savoir serait intériorisé par la pratique sans nécessité de verbalisation. Lors de la description de l'action rituelle Akiriket, j'ai tenté de mettre en évidence cet apprentissage progressif du savoir-faire rituel : les plus jeunes épient sans participer, les adolescents qui aident leurs aînés intériorisent par la pratique les gestes opportuns tandis que les aînés qui ont intériorisé le processus dans son entier se limitent à une supervision lâche de l'entreprise. Ces savoirs influencent directement l'agent (sans qu'il le sache explicitement) dans ses interactions avec les autres et dans son appréhension des événements auxquels il assiste ou participe.

\section{L'ATTITUDE RITUELLE}

Un autre type de capacité est particulièrement intéressant pour mon propos. Il s'agit de ce que J. R. Searle désigne comme les structures causales (Searle 1995 : 169). En tant qu'être humain, nous avons la capacité intuitive de discerner des causes et des effets. Cette capacité à relier des phénomènes dans des schèmes causaux est pour une bonne part issue de la phylogenèse de l'espèce humaine. Mais cette capacité s'exprime dans des contextes culturels différenciés. L'action rituelle est l'un de ces contextes, dans lequel la relation causale a un statut particulier. Dans l'Ekimomwar et, plus généralement, dans l'ensemble des rituels turkana, les acteurs semblent accepter le fait que par une action sur le corps de la société (dans la situation présente, la « remise en ordre » de la société), il est possible d'agir à un tout autre niveau (la « remise en ordre » du monde). Cette croyance, issue de capacités d'arrière-plan, n'est pas explicitée par les membres de la population mais elle est nécessaire pour que l'action rituelle puisse exister. Sans la croyance implicite en l'efficacité de la procédure, celleci aurait rapidement disparu.

Cette disposition à accepter l'efficacité du rituel sur base de l'expérience d'autrui (ou de l'expertise de ceux qui ont déjà agi) fait donc partie de l'arrière-plan culturel turkana. La répétition des rituels dans des situations déterminées rend possible l'acquisition de cette capacité pour les membres de la société. Ils se familiarisent avec la procédure dès le plus jeune âge. Le mode d'action rituel devient ainsi la réponse conven-tionnellement adéquate pour des situations spécifiques (i.e. pour lesquelles l'action a été exécutée par des prédécesseurs). Le processus répond désormais aux attentes générales et individuelles. En ce sens, je peux affirmer que le rituel intervient dans la constitution et la structuration de l'arrière-plan des individus mais qu'il en est aussi une conséquence.

\section{UN INVARIANT DES RITUELS : LEUR STRUCTURE SPATIO-TEMPORELLE}

Le processus décisionnel qui, il y a des générations, a été à l'origine des deux actions traditionnelles que nous avons analysées brièvement, nous restera toujours inaccessible. Il m'est, de ce fait, difficile de décider, entre la conceptualisation de 
la course du soleil (son anthropomorphisation) et celle du contexte écologique et social (les valeurs de la configuration des terroirs), celui des deux éléments qui fut déterminant pour l'émergence des modèles rituels turkana. Sans doute ont-ils tous deux exercé des influences combinées, influences que les conceptions actuelles de ces réalités continuent à exercer, par ailleurs, sur les explicitations que les acteurs peuvent donner de leurs actes. Quoiqu'il en soit, dans l'univers pastoral turkana, l'axe kide/too est particulièrement saillant. D'une part, il s'agit du seul axe fixe, aisément repérable en tout point de l'espace, grâce au soleil toujours présent. D'autre part, c'est approximativement le long de cet axe que s'effectuent les transhumances.

Cette forte saillance en fait un expédient « économique » pour structurer l'acquisition et la mémorisation, par les membres de la société, d'informations de statuts divers. L'analyse révèle, au minimum, trois modes de fonctionnement de l'indice.

En première analyse, l'indice matériel (notre axe et les deux pôles qu'il détermine) constitue un point de référence. Il permet la spatialisation des phases de l'action. En ce sens, l'indice est un schème spatio-temporel. Il est nécessaire à l'installation, au maintien et à l'organisation de savoir-faire et de compétences (rituels ou non) dans la mémoire procédurale des acteurs. En effet, du point de vue de ces derniers, les différentes composantes matérielles de l'aire rituelle n'existent que par rapport à cet axe (i.e. elles sont directement et toujours orientées). Cette configuration spatiale constitue une matrice qui permet, et d'une certaine manière entraîne, des configurations relationnelles spécifiques (i.e. les différentes catégories sociales occupent des postes spécifiques). Ces configurations rituelles favorisent, à leur tour, l'intériorisation de modèles particuliers de comportement (qui ne seront pas exclusivement réservés au rituel) comme, par exemple, les différents modèles d'interaction entre aînés et cadets et les modèles adéquats d'expression de l'individualité.

En deuxième analyse, l'indice matériel et la configuration rituelle dans laquelle il est immergé supportent la mémoire sémantique de l'observateur. Ils interviennent dans la constitution, la stabilisation et la réactivation périodique de ce que j'appellerai, à la suite de Daniel Schacter, de vastes réseaux d'associations et de concepts qui soustendent notre connaissance générale du monde (Schacter 1999 : 166). En d'autres termes, ils induisent les observateurs à associer des idées acquises dans différents contextes. Dans mes développements, j'ai mis en évidence, à diverses reprises, des réseaux d'association pour les deux pôles. Too était globalement connoté négativement dans le quotidien pastoral et dans la temporalité du rituel. Kide, à l'inverse, était connoté positivement. Ainsi, lors de mes interviews, pendant le rituel de l'Ekimomwar, mes informateurs recouraient indistinctement à des éléments de la logique rituelle générale turkana («c'est comme la akiriket, orientée vers l'est, c'est la règle ») ou de la quotidienneté ( « c'est le lieu des ennemis, c'est dangereux ») pour justifier le choix des pôles négatif et positif de la procédure rituelle d'évitement de l'infortune.

Je crois aussi, plus généralement, que ces indices (non naturels et intégrés dans les rituels) sont des déclencheurs de l'interprétation (dont l'explicitation reste, cependant, facultative). Alfred Gell parle d'une certaine forme d'indéchiffrabilité fondamentale de ce type d'indice en ce sens que le sujet percevant n'a pas la capacité de reconstruire dans son ensemble les processus mentaux qui sont à l'origine de l'indice (Gell 1998 : 71). Le destinataire peut uniquement s'adonner à une reconstruction du sens de l'objet visuel en vertu de la contiguïté ou de la ressemblance (quand l'indice a des aspects iconiques) avec ce qu'il désigne et d'informations partielles éventuellement 
transmises. Cette recherche de sens peut aussi fort bien se limiter à une reconnaissance du bien fondé de la création de l'indice (i.e. il a été produit par des personnes qui savaient). La sous-détermination de l'indice fait sa force et explique, en partie, son maintien dans la longue durée. Il présente une sorte d'évidence dans un contexte historique et culturel mais il reste ré-interprétable et réactua-lisable lors des modifications progressives de ce contexte.

En troisième et dernière analyse, le schème spatio-temporel structure les épisodes spécifiques de la vie d'un individu. Des événements a priori perçus comme problématiques, un désordre biologique par exemple, sont repensés en termes prototypiques : le dualisme de toute chose et l'oscillation que ce dualisme implique. Mais, plus fondamentalement, à un niveau pré-épistémique, ce schème agit sur l'état mental des agents. Chez l'individu trop marqué par la charge émotionnelle d'événements récents (infortune, meurtre, raid, etc...), il remodèle les états mentaux et permet ainsi la réinsertion dans un contexte social « ordinaire ». Le rituel façonne donc directement la conscience qu'un individu a de lui-même. Ces états mentaux, en constante évolution, ponctuellement « redéfinis » grâce à des procédures rituelles, conditionne à leur tour les expériences futures de l'individu. L'itération entre rituel et événements marquants de la vie renforce ainsi progressivement une esthétique particulière (i.e. une manière de ressentir le monde).

Mon problème de ne pouvoir accéder à la (aux) pensée(s) source(s) de l'action traditionnelle est aussi celui de tout Turkana. Nous l'avons vu auparavant, la tradition est muette sur le processus décisionnel de départ. Par contre, ce qui se transmet d'exécution en exécution sont les propriétés formelles de l'action. Evidemment, des rationalisations et interprétations sont passées d'une génération à l'autre. Mais, étant donné le changement de contexte historique, leur valeur sémantique a très certainement dû évoluer. Ainsi, un enfant naît dans un contexte et participe à des processus qui ont été « constitués » par ses prédécesseurs. Lui-même, à son tour, participera à la transmission de ces éléments culturels à la génération suivante et, avec ses contemporains, il interviendra dans la transformation progressive de cette tradition.

Dès le début, l'enfant est donc immergé dans deux types de spatialisation (rituelle et pastorale) dont nous avons vu comment elles se faisaient écho. Les représentations rituelles de l'espace ainsi que le rapport pastoral à cet espace influencent son appréhension globale de la topographie. Les attitudes et dispositions qu'il développe ainsi pourront ou non donner lieu à des interprétations de sa part, lorsqu'il s'efforce de traduire en mots (de rationaliser) ce qu'il ressent. Je crois que c'est ce modelage des affects qui est au cœur du fonctionnement de la tradition. En ce sens, la procédure rituelle est un moyen particulièrement efficace et économique pour réitérer l'expérience des générations antérieures même si ce processus reste toujours ré-interprétable dans certaines limites. 


\section{RÉFÉRENCES BIBLIOGRAPHIQUES}

BERQUE A.

1995 Les raisons du paysage : de la Chine antique aux environnements de synthèse, Paris : Ed. Hazan : $192 \mathrm{p}$.

BLOCH M.

1997 La violence du religieux, Paris : Ed. Odile Jacob : 225 p.

BOYER P.

1990 Tradition as truth and communication : a cognitive description of traditional discourse, Cambridge : Cambridge University Press : 143 p.

1997 La religion comme phénomène naturel, Paris : Bayard Editions : 333 p.

\section{COSTERMANS J.}

$2001^{2}$ Les activités cognitives : raisonnement, décision et résolution de problèmes, Bruxelles : Ed. De Boeck Université : 239 p.

DAMASIO A.R.

1999 Le sentiment même de soi : corps, émotions, conscience, Paris : Ed. Odile Jacob : 380 p.

DIMENDAAL G.J.

1983 The Turkana Language, Dordrecht : Foris Publications : 496 p.

GELL A.

1998 Art and Agency : towards a new anthropological theory, Oxford : Clarendon Press : 271 p.

GULLIVER P.H.

1951 A preliminary survey of the Turkana : a report compiled for the Government of Kenya, Communications from the School of African Studies : new series $n^{\circ} 26$, Cape Town : University of Cape Town : $281 \mathrm{p}$.

1955 The family herds : a study of two pastoral tribes in East Africa, the Jie and Turkana, London : Routledge \& Kegan Paul Ltd : 271 p.

1958 The Turkana Age Organisation, American Anthropologist 60/5 : 900-922.

HOUSEMAN M., SEVERI C.

1994 Naven ou le donner à voir : essai d'interprétation de l'action rituelle, Paris : CNRS Editions : $224 \mathrm{p}$.

LIENARD P.

2003 (n.p.) Le comportement rituel : communication, cognition et action. Génération, âge, filiation et territoire : contribution à l'ethnographie de deux populations du Cercle Karimojong (les Turkana du Kenya et les Nyangatom d'Ethiopie), thèse présentée en vue de l'obtention du grade de Docteur en Sciences Sociales, orientation Anthropologie Sociale et Culturelle.

\section{MÜLLER H.K.}

1989 Changing Generations and Age-Sets in Southeastern Sudan (Toposa) and Northwestern Kenya (Turkana), Saarbrücken - Fort Lauderdale : Verlag Breitenbach Publishers : 204 p. (Spektrum 17).

1991 Generation Sets : Stability and Change with Special References to Toposa and Turkana Societies, Bulletin of the School of Oriental and African Studies 54/3 : 554-567.

\section{OHTA I.}

1989 A classified vocabulary of the Turkana in northwestern Kenya, African Study Monographs, Supplementary Issue $10: 1-104$.

PELLETIER J.

2000 Voir un fictum dans une image, in LIVET P. (dir.), De la perception à l'action : Contenus perceptifs et perception de l'action, Paris : Librairie Philosophique J. Vrin : 155-190. 
SCHACTER D.L.

1999 A la recherche de la mémoire : le passé, l'esprit et le cerveau, Paris-Bruxelles : Ed. De Boeck Université : 408 p.

SEARLE J.R.

1998 La construction de la réalité sociale, trad. de l'anglais par C. TIERCELIN, Paris : Ed. Gallimard 'nrf essais' : 306 p. 